\title{
Zur Differentialdiagnose zwischen Dementia praecox und Hysterie bzw. Psychogenie.
}

\author{
Von \\ Dr. Alfred Wichmann.
}

(Aus der Psychiatrischen und Nervenklinik zu Königsberg i. Pr. Direktor: Geh. Rat Prof. Dr. E. Me yer.)

(Eingegangen am 27. März 1921.)

Die Differentialdiagnose zwischen Hysterie und Dementia praecox, wichtig vor allem wegen des Unterschieds ihrer Prognose, ist trotz der Verschiedenheit ihrer Ätiologie und ihres Wesens keineswegs stets einfach. Beide Erkrankungen können symptomatologisch sich außerordentlich ähneln. Das hat auch J ung dazu veranlaßt, zwischen beiden Krankheiten eine psychologische Verwandtschaft (allerdings keine Identifikation) zu konstruieren, indem er, ähnlich wie Freud die Hysterie, so die Dementia praecox auf verdrängte Komplexe zurückführt, diesen also auch hier eine ätiologische Rolle zuteilt, nur mit dem Unterschiede, daß die Komplexe bei der Dementia praecox dauernd fixiert seien. Die J ungsche Auffassung wird ziemlich allgemein abgelehnt, unter anderen von Isserlin, der die Methodik als.unwissenschaftlich angreift, und von E. Me yer, der darauf hinweist, daß die Komplexe nicht die Krankheit hervorrufen, sondern daß sie nur bestehen, weil die Krankheit ihnen gestattet, unkorrigiert hervorzutreten. Aber wie man sich auch zu der Komplexfrage stellt, sicher ist, daß Hysterie und Dementia praecox grundverschiedene Krankheiten darstellen vor allem in ihrer Affektivität. Es ist kein Zufall, daß die hysterischen Symptome bei der Dementia praecox namentlich zu Beginn auftreten, solange noch keine ausgesprochene gemütliche Verblödung besteht.

Die differentialdiagnostischen Schwierigkeiten liegen wie erwähnt darin, da $B$ die Symptome beider Krankheiten sich weitgehend gleichen können. Es gibt überhaupt wohl kaum ein Symptom, das für eine bestimmte Erkrankung pathognomonisch ist, vielmehr kommen die gleichen Symptome bei den verschiedenartigsten Krankheiten vor. So sei beispielsweise daran erinnert, daß katatone Erscheinungen bei fast allen Geisteskrankheiten beobachtet worden sind, ja, daß selbst ausgesprochene katatonische Zustandsbilder bei den verschiedenartigsten Psychosen (progressive Paralyse, Hirntumor, Arteriosklerose, degenera- 
tive Psychosen, usw.) gar nicht selten sind. Indessen bestehen wohl schließlich trotz aller Ähnlichkeit der Symptome doch feinere Unterschiede, die z. T. bereits bekannt, zum größten Teile aber noch unbekannt sind. Die vorhandenen Abgrenzungsversuche sind noch keineswegs sicher und unbestritten.

Im folgenden seien einige wichtige Symptome besprochen, die besonders oft Schwierigkeiten bei der Differentialdiagnose Dementia praecox und Hysterie bieten. Der Negativismus der Schizophrenen kann mit dem ablehnenden, schnippischen oder eigensinnigen, trotzigen, zornigen Wesen der Hysteriker verwechselt werden; Ausbleiben von Abwehrbewegungen oder Schmerzensäußerungen auf Nadelstiche kann auf Negativismus oder auf Anästhesie beruhen. Rhythmische Bewegungen, Stereotypien, Grimassieren kommt bei beiden Krankheiten vor. Im allgemeinen sind die Stereotypien der Dementia-praecox-Kranken triebartiger und unbeeinflußbarer. Aber Löwy weist darauf hin, daß die Stereotypien der Schizophrenen durchaus nicht so unbeeinflußbar und der Behandlung unzugänglich sind - vorübergehend natürlich -, als man gemeinhin annimmt. Befehlsautomatie kann mit Schüchternheit, sinnlose Verschrobenheiten der Schizophrenen können mit hysterischer Geziertheit verwechselt werden. Das manierierte, läppische, erotische Benehmen derDementia-praecox-Kranken kann dem kindlichen, koketten, gezierten, erotischen, hysterischen Gebaren gleichen. Erregungen, Wutausbrüche, läppische Erregungszustände finden sich bei beiden Krankheiten. Nach Krae peli n spielen sich die katatonischen Erregungen auf engerem Raume ab; im allgemeinen finden keine Angriffe auf die Umgebung statt, der Schlaf der Katatoniker ist selbst nach den heftigsten Erregungen gut. Auch sind die Katatoniker während der Erregungszustände völlig klar, während bei den affektiven Erregungen der Hysteriker das Bewußtsein getrübt zu sein pflegt. Der gleiche Unterschied soll im Stupor bestehen. Doch kann der Grad der hysterischen Bewußtseinstrübung sehr erheblich schwanken und auch im katatonischen Stupor kann nach Kr rae pelin eine, wenn auch nicht sehr erhebliehe Bewußtseinstrübung bestehen. In sehr seltenen Fällen können katatonische Krämpfe den hysterischen völlig gleichen.

Bewußtseinstrübung kann aber auch durch die Aufmerksamkeitsstörung der Schizophrenen vorgetäuscht werden. Verwirrtheitszustände haben bei beiden Krankheiten eine völlig verschiedene Grundlage, können sich aber in ihren äußeren Erscheinungen sehr gleichen. Auch hier sind die Hysterischen mehr oder weniger in ihrem Bewußtsein getrübt, es besteht meist ein Zusammenhang mit den Vorgängen der Umgebung oder gewissen Erlebnissen, es ist ein gewisser Sinn zu erkennen, man sieht oft einen Affekt z. B. Angst deutlich durchschimmern. Die Katatoniker urteilen bei ihrer Verwirrtheit doch klar, beobachten ungetrübt durch Affekte rịchtig, wenn auch bisweilen ungenau, geben zwischendurch völlig richtige und zutreffende Antworten. Andererseits kann aber auch in leichten Fällen schizophrener Sprachverwirrtheit eine oberflächliche assoziative Verknüpfung bei völliger Wahrung des Satzbaues vorhanden sein. Sinnloses Perseverieren findet sich auch bei Hysterie auf Grund einer Gemütsbewegung. Erscheinungen wie Agrammatismus, sinnloses Zeichnen und Kitzeln kann in der Deutung erhebliche Schwierigkeiten machen. Die Halluzinationen der Hysteriker finden sich namentlich auf optischem, die der Schizophrenen auf akustischem sowie auf dem Gebiet der allgemeinen Körperempfindungen. Übrigens sind die Halluzinationen der Hysteriker vielleicht gar keine echten Halluzinationen, sondern nur lebhafte Vorstellungen, hervorgerufen durch starken Affekt. So würde es sich auch erklären, daß sie mehr auf optischem Gebiet liegen, da wir uns optische Bilder leichter vorstellen können als Klangbilder und Körpersensationen. Den wechselnden, beeinflußbaren 
Mißempfindungen der Hysterischen stehen die hartnäckigen der Dementia-praecoxKranken gegenüber, die von diesen, wie Krae pelin hervorhebt, gern zu einem unsinnigen hypochondrischen oder Verfolgungswahn verarbeitet werden. Die Wahnbildung interessiert bei der Frage der Differentialdiagnose ganz besonders ${ }^{1}$ ). Birnbaum hat an Hand von 109 Fällen die Wahnbildung der Degenerierten zu charakterisieren und zu umgrenzen versucht. Sie seien oberflächlich verarbeitet, unbeständig, flüchtig, in ihrem Inhalt variabel und beeinflußbar, nicht systematisiert, mit unnützem Beiwerk versehen, entsprächen bestimmten Wünschen, seien in ihrem Entstehen und Schwinden von äußeren Anlässen abhängig. Aber erst auf Bleulers Einwände gab er die Unterschiede gegenüber der Dementia praecox an: Entstehung durch äußere affektiv wirksame Erlebnisse, schnelles Losbrechen und volle Entwicklung der Psychose, die zu Beginn oft von einer Bewußt. seinsstörung begleitet ist, Abhängigkeit des Verlaufs von äußeren Verhältnissen, Beziehung zwischen Erlebnis. und Wahninhalt, enge Beziehungen zu den hysterischen Psychosen. Entstehung durch äuBere affektive Anlässe und Abhängigkeit im Verlauf von äußeren Umständen sind indessen keine stichbaltigen Kriterien. lch komme hierauf später zurück. Krae pelin charakterisiert die Wahnideen der Schizophrenen als unsinniger, verblüffender, oberflächlicher verarbeitet, innerlich beziehungsloser, nur vorüborgehend systematisiert, später zerfahrener, widerspruchsvoller. Der hysterische Wahn soll infolge Fehlens einer Urteilslosigkeit. im allgemeinen mit der Bewußtseinstrübung schwinden ${ }^{2}$ ). Ein charakteristiseher Unterschied läßt sich nicht festlegen, die Beurteilung scheint mehr Gefühlssache zu sein.

Ich möchte nicht noch weitere differentialdiagnostische Schwierigkeiten aufzïhlen, sondern nur noch bemerken, daß gelegentlich einmal bei der Dementia praecox auch Symptome vorkommen, die sonst fast ausschließlich bei Hysterikern grefunden werden. So fand $\mathrm{B}$ accelli bei einem 19 jährigen Katatoniker hysterisches Luftschlucken, das auf Kommando unterbrochen und wieder hervorgerufen werden konnte, und in einem Falle von Schizophrenie von $\mathrm{H}$ albers tadt bestand 6 Jahre lang eine hysterische Paraplegie.

Zur Klärung der Differentialdiagnose kommen natürlich auch die körperlichen symptome in Betracht. Von den Pupillenstörungen differentialdiagnostisch brauchbar und für Dementia praecox einigermaßen pathognomonisch ist nur das Bu m kesche Phänomen: das Fehlen der Pupillenunruhe sowie der Psychoreflexe und der Reflexe auf sensible Reize. Mehr von theoretischem Interesse ist die Westph a lsche katatonische Pupillenstarre sowie das E. Me yersche Iliacalsymptom, welch letzteres von Westphal auch bei Hysterie nachgewiesen wurde. Fbenso ist die Adrenalinmydriasis nicht brauchbar; gerade in den zweifelhaften SchuItzschen Fällen versagte sic. Von den übrigen körperlichen Symptomen kann vielleicht noch die Abderhaldensche serologische Blutuntersuchung wie der Blutgerinnungsversuch herangezogen werden. Lähmungen, Contracturen, anästhetische Zonen, konzentrische Gesichtsfeldeinschränkungen sprechen im allgemeinen für Hysterie.

Die Differentialdiagnose kann nach alledem nicht allein durch Abgrenzung und Betrachtung der Symptome entschieden werden,

1) Auf den Lnterschied bzw. die Verwandtschaft der Hysterie mit den übrigen degenerativen Psychosen gehe ich nicht näher ein. Um so mehr dürfte es sich hier erübrigen, zu dieser Frage Stellung zu nehmen, als wir ja ganz allgemein versuchen müssen, die hysterischen, degenerativen sowie die Psychosen ohne jede degenerative Grundlage von der Schizophrenie abzugrenzen.

2) Dies läßt sich übrigens nicht so ohne weiteres auf alle übrigen degenerativen und sonstigen psychogenen Wahnbildungen übertragen. 
sondern es kommt mehr auf den Gesamteindruck des ganzen Zustandsbildes an. Aber auch die Zustandsbilder sind nicht immer einwandfrei zu deuten, da sie sich gleichfalls mit ihren Zutaten und in ihren Einzelheiten oft gleichen. Es sei hier auf die ausführliche Arbeit Sterns, sowie auf die Arbeiten von Bonhoeffer, Kaiser, Birnbaum, Lückerath, Kutner, Kreuser, Raecke, Loewenstein hingewiesen. Einerseits kann es bei der Hysterie zu Zustandsbildern kommen, die wir sonst bei der Schizophrenie sehen, andererseits können, wie erwähnt, bei einer Dementia praecox namentlich zu Beginn, aber auch im weiteren Verlauf, schließlich wie Halberstadt zeigt, auch in den Endzuständen hysterische bzw. hysteroide Zustandsbilder auftreten. Die Fälle der obenerwähnten Autoren zeigen, dàß es unmöglich sein kann, aus dem Querschnitt der Psychose eine sichere Diagnose zu stellen. Oft muß die Anamnese, der Verlauf, der Endausgang, die Katamnese zu Hilfe genommen werden, um zur Diagnose zu gelangen.

Differentialdiagnostische Schwierigkeiten bereiten besonders die hysterischen und degenerativen Situationspsychosen. Vor dem Krieg standen hier im Vordergrund die sog. Haftpsychosen. Kutner, Stern und Risch sahen als Kriterium für ihre psychogene Natur die Entstehung durch affektive Erlebnisse sowie die Beeinflussung durch äußere Einflüsse. Aschaffenburg hebt hervor, daß weit mehr als gewöhnlich angenommen die Psychosen in der Haft nur Exacerbationen bzw. die ersten deutlichen Symptome der Dementia praecox darstellen. Raecke betont, daß der Einfluß der Situation und die Abhängigkeit der Psychose in ihrem weiteren Verlauf von äußern Umständen sehr überschätzt werde. Gestützt auf Erfahrungen im Kriege, wo die Mannigfaltigkeit der auslösenden Ursachen - Granatexplosionen, Fliegerangriffe usw. - und damit der Situationspsychosen viel größer war, hat E. Me yer darauf hingewiesen, daß der Nachweis eines stark affektbetonten Erlebnisses differentialdiagnostisch keine Bedeutung habe, daß vielmehr die Diagnose sich auf die Krankheitsbilder selber stützen müsse. Es können auch bei der Dementia praecox psychogene Symptomenkomplexe auftreten, welche durch die Situation hervorgerufen und erhalten werden. Sch neider und Friedlä nder gehen noch weiter. Bisher nahm man an, daß ohne dies affektive Erlebnis die Dementia praecox doch ausgebrochen wäre. Es bestanden vielleicht vorher schon leichte Erscheinungen, durch die Situation bekam die Krankheit nur ihre (psychogene) Färbung. Schneider und Friedländer glauben auf Grund ihrer Erfahrungen im Kriege, daß in manchen Fällen die Dementia praecox durch affektive Fronterlebnisse direkt ,ausgelöst", „verursacht" würde. Nach dem allen ist jedenfalls sicher, daß wir bei der Differentialdiagnose auf Situation und Beeinflussung durch äußere Verhältnisse kein entscheidendes Gewicht legen dürfen. Damit kommt 
ein weiteres, früher wichtiges differentialdiagnostisches Mittel in Wegfall.

Und noch etwas hat der Krieg gelehrt, ganz abgesehen davon, daß vielleicht gewisse Zustandsbilder häufiger wurden und öfters Veranlassung zu differentialdiagnostischen. Schwierigkeiten gaben, wie etwa delirante Zustände, Puerilismus, Pseudodemenz (vgl. die Arbeiten von Popper, Bonhoeffer usw). Vor dem Kriege nahm man an, daß in fraglichen Fällen es sich eher um Dementia praecox als um Hysterie handele. So hält Nissl z. B. hysterische Psychosen direkt für eine Seltenheit und Kraepelin gibt an, daß man in dubio eher eine Dementia praecox als eine Hysterie annehmen solle. Im Kriege hat sich das geändert. Popper, Schmidt u. a. kamen zu der Überzeugung, daß man viel zu viel Dementia praecox diagnostiziere.

Noch eine weitere Frage sei kurz berührt, da sie für die Differentialdiagnose von Bedeutung ist, nämlich die Frage nach hysterischen Begleitsymptomen der Dementia praecox. Die meisten Autoren lehnen es ab, diese mit den echten hysterischen Erscheinungen zu identifizieren, sondern sprechen nur von ,hysteriformen", ,hysteroiden" Symptomen. Da aber solche Symptome namentlich zu Beginn der Dementia praecox als erste Manifestation der Geisteskrankheit auftreten, so ergibt sich die Frage, ob die beiden Krankheiten sich kombinieren können, d. h. ob sich zu einer echten, genuinen Hysterie eine Dementia praecox gesellen (Kaiser, Nissl, Kreuser und Gaupp nehmen dies an), oder ob eine Dementia praecox eine Hysterie hervorrufen kann. Es würde sich dann um eine symptomatische Hysterie (Kraepelin) handeln. Kreuser gibt zur Mlustration dieser Frage drei Dementiapraecox-Fälle, die hysterische Symptome in seltener Reinheit zeigen. Bejaht man die Frage, so muß man mit Kreuser folgenden Schluß ziehen: Im Zweifelsfalle haben nur die schizophrenen Symptome Wert, hysterische Erscheinungen berechtigen nicht zur Annahme einer Hysterie, sie können auch symptomatische sein, oder es kann sich auf sie eine Dementia praecox aufgepfropft haben.

Es wurde bereits erwähnt, daß aus dem Querschnitt der Psychose die Diagnose oft nicht zu stellen ist. Aber auch der Längsschnitt, der Verlauf bringt nicht in allen Fällen Klärung. So bei Lückeraths Fällen, bei einem Teil der Sternschen Fälle, Wachsmuths fünftem und ebenso dem Dreyfu sschen Fall. Selbst durch Monate fortgesetzte eingehende Beobachtung gibt zuweilen kein anderes Bild, als es die Querschnittsbetrachtung bot, und erlaubt somit nicht immer ein abschließendes Urteil. Als Beweis und Beispiel teile ich nachstehend einen Fall mit, der nunmehr schon seit 12 Monaten in Beobachtung steht. 


\section{Vorgeschichte.}

Fiedrich Priedrigkeit, am 24. XI. 1892 zu Raumo bei Senftenberg, Kreis Calau in Brandenburg geboren; hat 3 Brüder und 2 jüngere Schwestern, die geistig und körperlich gesund sind. Die Brüder haben aktiv gedient und den Feldzug von Anfang bis zu Ende mitgemacht. Die ältere Schwester ist verheiratet, die jüngere übt ihren Beruf als Klavierlehrerin aus. Nach eigenen Angaben hat Pat. auf der Schule schwer gelernt; nach Mitteilung des Vaters die Schule regelmäßig besucht, etwas beschränkt, aus der 2. Klasse entlassen. Fleiß und Leistungen nach den Sohulzeugnissen, ,genügend". Nach der Schulentlassung arbeitete P. in Hamm, dem Wohnsitze seines Vaters, auf der Zeche als Lehrhauer 6 Jahro lang. Nie krank gewesen. Lebenswandel einwandfrei.

Am 1. X1. 16 zum E.-Inf.-Reg. 131, Bielefeld, Bez.-Kommando Soest eingezogen, 2 Monate ausgebildet und (wahrscheinlich auf Reklamation) zur Arbeit entlassen. 1917 zum 2. Male einberufen und am 21. I. 18 wiederum beim E.- Inf.R. I31 eingestellt. Am 21. III. 18 aus unbekannten Gründen ins Vereinslazarett Bethel eingeliefert und von dort am 20. IV. 18 nach Heil- und Pflegeanstalt Lengerich überführt. In dem Überweisungszengnis aus der Anstalt Bethel heißt es: „Pr. ist am 21. III. 1918 in Bethel eingeliefert. Alle näheren Angaben fehlen bisher. Pr. selber weiß nicht, weshalb er hierher gekommen ist. Er macht den Eindruck eines etwas schwachsinnigen Menschen."

In Lengerich gibt P. an: 3 Brüder und 2 Schwestern sowie Mutter gesund, Vater schon kränklich, hätte Lungenentzündung gehabt, arbeitete auf Zeche Sachsen, wo P. auch die letzten 6 Jahre als Lehrhauer gearbeitet habe bei $6,50 \mathrm{M}$ Tagesverdienst. Als Kind nicht krank gewesen. Finmal vor 6 Jahren, 1911, habe er os an der Lunge gehabt, sei 8 Monate im kath. Krankenhause in Hamm i. Westf. gewesen. Vor ungefähr 4 Wochen beim Militär wegen Halsschmerzen krank gemeldet, deshalb nach Bethel; habe nicht gut schlafen, aber gut essen können. Habe jetzt noch Schmerzen in der Halsseite und Seitenstechen.

Die psychische Prüfung ergab am 21. IV. 18: P. weiß, wo er sich befindet, kennt aber nicht die genauere geographische Lage des Ortes, weiß nicht, wie er nach Lengerich gefahren ist. Die Daten aus seiner Militärzeit gibt er genau und richtig an. Rechnen, besonders Dividieren sehr schlecht. (Kaiser?) Wilhelm II. (Regiert wie lange?) Jetzt 68 Jahre, 68 Jahre hat er regiert. (Alter?) 60. (Sein Vater?) Weiß ich nicht. (Krieg?) Jetzt 4 Jahre. (Anfang?) 1914. (Monat?) 2. VIII. (Mit Rußland?) Ist jetzt etwas stille. (Wie lange?) Im Mïrz. (Andere Feinde?) England, Frankreieh, Griechenland, Japan, Portugal, Rumänien, das alles. (Wie mit Rußland?) Konnten nicht weiter die Russen. (Himmelsrichtungen?) Sonne geht im Westen auf, im Norden unter. (1 Jahr Tage?) 136. (1 Jahr Wochen ?) 112. (1 Monat Tage?) 240. (1 Monat Tage?) 31. (240 Tage wieviel Monate?) 6 Monate. (1 Monat wieviel Tage?) 31. (6 Monate wieviel?) 6. $31=181$. (240 Tage wieviel Monate?) 6 Monate. (Vaterunser?) † (Glaubensbekenntnis ?) Trotz Hilfen - (5. Gebot?) Sollst nicht stehlen. (Was wissen Sie von Jesus Christus ?) Ist auferstanden von den Toten. (Wann?) Im März. (Wie lange her?) 1 Monat 3 Tage. (Wann Ostern?) 22 Tage her. (Was da gefeiert?) - +. (Was Pfingsten?) Christi Himmelfahrt. (Weihnachten?) Geburt Jesi Christi. (Wo gehoren ?) Bethlehem. (Wo liegt das?) Weiß ich nicht. (Wann geboren ?) 1917 (nach Besinnen). (Wann gestorben?) Weiß ich nicht. (Wie alt geworden?) 197 Jahre. (Woher wissen Sie das?) Weil wir jetzt 1917 haben. (Wo hier?) +. (Haus?) Haus Lengerich. (Was für Leute hier?) Brüder. (Warum?) weil sie aufpassen müssen, daß einer keine Anfälle kriegt. (Bestraft?) Noch nicht.

26. VI. 18. Albernes Benehmen. Bei Versuchen, ihn zur Arbeit mitzunehmen, läuft er ziellos umher; weiß nicht, worum es sich handelt. Beim Spaziergang ver- 
lief er sich einmal und wurde unterwegs gefunden, wo er sich sehr leicht hätte zurückfinden und zurückfragen können.

Status somaticus: Ernährungszustand gut. Größe $167 \mathrm{~cm}$. Ziemlich kräftiger Körperbau. Große Ohren, breiter Mund, gewulstete Lippen, breiter Gaumen, breite Nase, Schielen nach innen namentlich links, niedrige Stirn. Auf dem Kopfe 7 oder 8 Narben, angeblich von einer Schlägerei herriuhrend (1913). Lungen: hinten leichtes Giemen. Sonst innere Organe o. B. Gesichtsausdruck matt. Pupillen reagieren auf L., gleichweit. Reflexe + , nur Conjunctivalreflex 0, Patellarreflexe ++ . Ovarie 0. Sprache matt, tonlos. Haltung, Gang etwas steif. Sensibilität: Schmerzempfindung + herabgesetzt, Berührungsempfindung + , Unterschied von spitz und stumpf nicht regelmäßig bezeichnet. Sehschärfe r. 5/5, 1. 5/7,5. Gehör + . Sonst Nervensystem und Sinnesorgane o. B. Diagnose: angeborener Schwachsinn. Deswegen am 20. X. 18 aus dem aktiven Heeresdienst entlassen. Nach Angaben seines Vaters klagte P. um diese Zeit über ,Nervenleiden" und "Nervenzittern". Im März 1919 verließ er den Wohnort seiner Eltern, ging nach Schwerte i. Westf., um leichtere Arbeit zu finden. Seither nicht mehr nach Hause geschrieben. Über die Zeit von März bis Ende Oktober 1919 nichts Sicheres bekannt.

Am 1. XI. 19 erschien P. im Durchgangslager Heilsberg, gab an, Heimkehrer aus der Kriegsgefangenschaft zu sein, ,offenbar um sich die Vorteile eines heimgekehrten Kriegsgefangenen (Entlassungsgeld und Entlassungsanzug) anzueignen". (Meldung des dortigen Gerichtsoffiziers.) Da man Verdacht schöpfte, wurde Untersuchungsverfahren wegen Betrugs eingeleitet.

Dem Gerichtsoffizier gab Pr. am 6. XI. 19 folgendes an: „Ich heiße Johann Brüdigkeit, bin 27 Jahre alt, kath. Glaubens, Sohn des verstorbenen Landwirts Georg Brüdigkeit in Ostpreußen. Ich bin am 24. XI. 1892 in Raunau (Kreis Heilsberg) geboren. Wo ich die Schule besucht habe, weiß ich nicht mehr. Vor dem Kriege arbeitete ich bei dem Besitzer Hennig in Raunau. Im Februar 1915 wurde ich eingezogen und kam zum. Inf.-Reg. 54. Im November $1915 \mathrm{kam}$ ich ins Feld nach Rußland und wurde im Februar 1917 in Brest-Litowsk gefangen. Mein Kompagnieführer hieß Hautpmann Schröder. In Rußland arbeitete ich in den Bleiwerken. Im März 1918 entfloh ich aus der Gefangenschaft und kam über Österreich, Frankreich und Deutschland. In Luxemburg kam ich über die deutsche Grenze (im März 1919). Ich kam ins Lazarett in Koblenz und nach 3 Wochen nach Altona. Von dort wurde ich dem Durchgangslager Heilsberg überwiesen."

Da die Angaben des P. nicht stimmten, wurde noch die Krankenschwester vom Durchgangslager Heilsberg vernommen. Sie gab unter anderem an: Br. sei am 1. XI. $19 \mathrm{im}$ Durchgangslager erschienen und durch die Äußerung aufgefallen, er wolle alle totschlagen, er bekäme für jeden von der Regierung $3 \mathrm{Mk}$. Vor seiner Aufnahme ins Lazarett habe er viel Unsinn geredet, aber immer dieselben Aus. sagen gemacht. Seinen Kameraden sei aufgefallen, daß er kein Kriegsgefangener sei, er habe nie etwas über die Gefangenschaft erzählt, wohl aber müsse er Soldat sein, da er alle Fertigkeiten eines Soldaten besäße. Ihr habe Br. erzählt, er sei in Koblenz im Lazarett gewesen und dort in nasse Tücher eingewickelt worden. Br. habe manche süddeutschen Worte gebraucht. Er habe erklärt, keine Angehörigen mehr zu besitzen; später habe er gesagt, er hätte eine Tochter namens Gretchen. Einmal wäre er auf die Frage der Heimkehrer hereingefallen. Er behauptete nämlich Brauweiler (im Rhld.) zu kennen. In Brauweiler sei aber eine Art Fürsorgeanstalt. Sie nehme an, Br. sei dort gewesen. Ferner habe er sich einmal vor dem Photographieren gesträubt; er ließe sich nur mit Bart photographieren, man solle ihm einen solchen verschaffen. Auch sei ihr aufgefallen, daß $\mathrm{Br}$, wenn er sich bei einem Kreuzverhör in seinen Antworten verwickelt hatte, das 
Gespräch sofort abgebrochen habe. Wegen Verdacht auf Nervenkrankheit wurde Br. der Nervenklinik Königsberg überwiesen.

Status und Verlauf. 16. XI. 19. Als Untersuchungsgefangener von 2 Soldaten aus Heilsberg gebracht. Bleibt ruhig im Bett. (Na me ?) Johann Brüdigkeit. (Datum?) 24. X. 1918. ('Tag?) Mittwoch. (Wo hier?) +. Angeblich mit 8 Jahren zur Schule, bis zum 14. J., schwer gelernt. Dann an 3 oder 4 Stellen gearbeitet. 1915 eingezogen beim I.-Reg. 132. War im Felde $1917 \mathrm{im}$ Westen gefangengenommen. Habe im Gefangenenlager in Ypern gesessen. Lief weg, war 8 Wochen unterwegs, hatte angeblich Proviant für die ganze Zeit mit. Meldete sich in Koblenz, kam von da nach dem Durchgangslager in Heilsberg. Hier in der Gegend sei er zu Hause. Ist 24 Jahre alt. Seine Frau sei im Kriege gestorben. Fin Kind von 6 Jahren, die Grete, lebt. Habe mit 23 Jahren geheiratet. Gibt jetzt sein Alter auf $28 \mathrm{~J}$. an. Niemals Gefängnis oder Arbeitshaus. Sei geisteskrank und deshalb hierher geschickt. In Heilsberg bezweifelten sie, daß er Kriegsgefangener sei. $2 \cdot 2=5 ; 2+4=2$. (Farbe des Schnees) blau. (Kennen Sie die Schwester?) Nein. (Das ist doch ein Mann?) Ja. Verlangt Sonntags ausgehen zu dürfen. Will morgen wieder weg. Draußen sei jetzt Sommer. Körperlicher Befund: Innere Organe o. B. Degenerationszeichen: Unterlippe stark gewulstet. Strabismus convergens, bes. rechts. Pupillen reagieren prompt. Conj.- u. Cornealreflex + . Rachenreflex scbwach + , Gaumenreflex + . Sehnen- u. Hautreflexe + . Patellarreflexe ++ . Babinski --. Kein Klonus. Sensibilität: Berührung + , Schmerz --, 'Tiefensensibilität + .

Die ersten Stunden nach der Aufnahme ruhig. Des Abends begann er viel zu erzählen, fing an zu renommieren, forderto die Wärter zum Ringkampf auf.

17. XI. 19. Kommt ruhig ins Untersuchungszimmer. Setzt sich auf den Stuhl, die Hände gefaltet. Schr bewegungsarm. Mund geöffnet. Verzieht keine Miene, bewegt nur die Augen. Sieht gleichgültig, zufrieden aus. (Name?) Johann Br. (Wie alt?) Werde jetzt 27, diesen Monat. (Geboren?) 24. XI. 92. ('Tag?) Mittwoch. (Datum ?) 21. X. 1918. Auf den Widerspruch aufmerksam gemacht, sagt er, er muß das sagen, wenn ihm das in den Kopf kommt. Weiß genau, da $B$ sein Geburtstag am nächsten Montag ist. (Wie lange hier?) Gestern, vom Durchgangslager Heilsberg. (Warum?) Nervenkrank, bin nicht richtig im Kopf. Sie haben mich schon eingesperrt, hat auch nichts genützt. Sie haben mich auch mit der Mütze photographiert. (Stadt?) +. (Haus?) +. (Gesund ?) Bin krank. Hatte schon Wutanfall in Gefangenschaft, brachten mich in Nervenanstalt Chepanie.

Schriftliche Beantwortung der Fragebogen.

I. (Name ?) Brüdigkeit. (Beruf ?) Landwirt. (Alter ?) 27. (Geboren ?) 24. XI. 1892. (Wo?) Rauno bei Sempftenberg. (Name der Eltern?) Luissi Brüdigkeit. (Was ist Ihr Vater?) August Brüdigkeit Landwirt. (Verheiratet?) Wittmann. (Wann geheiratet?) 25 Jahre. (Wie alt bei der Heirat?) 5 Jahre. (Wieviel Kinder?) V. (Gestorben?) 3 Kinder. (Name der Kinder?) Friedrich, Georg, Otte, 1I. Mächdel. (Alter der Kinder?) 28. 29. (Wo zur Schule ?) Hessen, Huckarte, Sehmidhorst. (Name der Lehrer?) Blume, Kettler, Olruge. (Name der Mitschüler?) Franz Rishahn, Mädhel, Pradmüller. (Wieviel Klassen durchgemacht?) 6. Sitzen geblieben?) Nein. (Welche Fächer leicht, welche schwer?) Alerlesche, Rechnen. (Nach der Schule?) Arbeiteten. (Welches Jahr?) 1919. (Monat?) November. (Datum?) 22. (Wochentag?) Dienstag. (Wie lange hicr?) 2 Tage. (Vor 8 Tagen ?) Heilsberg. (Wo gewohnt?) Raunau. (Wo vor einem Monat?) 12 Monat. (Wo Weihnachten?) 25 Dezember. Gefangen. Uber Zeitrechnung und Zeiteinteilung gibt Pat. richtig Auskunft. (Wann Weihnachten ?) 25. Dezember. (Wann Ostern ?) -. (Wann Pfingsten?) -. (Sommerbeginn?) 15. Juni. (Frühlingsanfang?) 21. März. (Herbstbeginn?) 15. Sempber. (Jetzt welche Jahreszeit?) Ssomer. (Hier 
welche Stadt?) +. (Haus?) +. (Wer hat Sie hergebracht?) - . (Was für Leute im Saale?) Kranke. (Name des Pflegers?) -. (Name der Ärzte?) -. (Krank ?) Nerzschut. Engelliche. (Traurig?) Ja. (Stimmenhören?) Sozialdemokrat. (Gestaltensehen?) Rot scharz. (Verfolgt?) Ija.

II. Rechenaufgaben werden mit Ausnahme einiger schwererer richtig gelöst. Etwas schwierigere eingekleidete Rechenaufgaben werden fast ausnahmslos nicht oder nicht richtig gelöst.

III. Geographische Begriffe völlig ungenügend, auch politische Begriffe sehr mangelhaft. Relativ gute Religionskenntnisse.

Schrift äußerst unbeholfen mit zahlreichen orthographischen Fehlern sowie Auslassungen von Buchstaben mit einzelnen Korrekturen. Manchmal wird der große Anfangsbuchstabe klein noch einmal wiederholt: Ssomer, Jja.

Le be nslauf. Lenbendlaug. Johann Brüdigkeit geboren 24. Noverber Rauno Semptenberg Kreis Kalau 1892. $8 \mathrm{Jahr}$ bin in der Schule gegangen. Da zuerst Hessen. Da ich ungefer $4 \mathrm{Jahr}$. Ich wieder verzugen nach Hinterte $1 \mathrm{Jahr}$. Und dach ich nach Rahm (?) im Schmidthorst. Dach das ist der aus der Schule. Das erst arbeit Hamborn. Zeche deusser Kaiser. Und d ... (?) Landwirt 3 Raunaus Ostpreußen. Das bin Artnnau v Kureland. Das ich nach Fraikhorst (?). Das leste 1 Jahr Raunau Heilsberg Hennig als Taglöhler. Das war ich Hereredienst 1915 Rekrut eingezogen. 1 Reg. 132 II Inftrie nach Straßburg. Da... ist ins Feld zurück Iich ... (?) Etag... (?)

18. XI. 19. In Rauno, Kreis Kalau geboren. Drei Brüder gefallen. Alle Angaben widersprechend. Darauf aufmerksam gemacht, sagt er: ,Ich kann nichts dafür, es ist nun mal im Kopf so." Behauptet, er heiße gar nicht Johann Brüdigkeit. Der Name sei ihm gekommen, und so nannte er ihn. Er heiße wirklich Franz Wiadrowski und sei in Bromberg geboren - . Haut sich vor den Kopf und fragt, warum er nicht loskäme.

19. XI. 19. Morgens recht unruhig und laut. Nach Spritze rubiger. Nachmittags schimpft er viol. Des Nachts warf er sein Bett heraus, sagte, wenn er nicht aus der Klinik entlassen wird, passiert etwas.

20. XI. 19. Schrie und sang vicl. Auf die Frage, warum er das täte, antwortoto er: „Ja ich möchte auch gern einmal eingepackt werden." Schimpft viel auf Ärzte und Schwestern.

21. XI. 19. Seinen Lebenslauf schreibt er jetzt wie folgt: Lenbens Bauf. Johann Brüdigkeit geborn 24. Nowember 1892. Sacd Pra Kurrieba Kascho Lonnka Beroniki Jäger matit Sa. Mein Lebenslauf heißt Ich heiß so und so ich est Vi ur. In inder Schule Hi eskö Brobewitz Prowienatz aus der Schule gekomme erst als Landwirt III Jahr. Als Und Lonas 1 Lnd bin ich Marnen 4 Monat Landwirt Da. bin Katogienas Zientraka Lascherschnnre Schadbrunn. Da hab Raunau Hennich habe ich arberdag 600 Das bin Sodat 1915 und 16.

22. XI. 19. Schreibt: Friedrich Priedrigkeit Nervenleiden schwach Murrenleiden Leibten Lungenkatra Keinen Stuhlgang Ein Soldat hat mit Gift gegeben Kochsalz, an das hat gelegen Gehirn erweiterung (durchstrichen) Herzleiden Am Iekose hat ich vill Kass geschlunt Von Schwester eine Schürze bekommen die war gut das erste Auge ist der sehen nerven stark Lnd habe Wasserbruch 20 Stärzen habe ich bekommen ich will lieder laden Und habe starkstrom Nervenschuck bekommen.

22. XI. 19. Abends sehr laut, sang und führte Selbstgespräche.

24. X1. 19. Macht dauernd Iärm, hetzt die andern auf, spricht dauernd im witzelnden Ton; Gesichtsausdruck listig und schlau. Tonfall beim Sprechen immer gleich; durch nichts wird P. aus der Ruhe gebracht. Auf jede Frage antwortet 
er, wie er gerade Lust hat. Wird ihm dies vorgehalten. so zuckt er mit den Achseln und meint, das müsse so sein.

25. XI. 19. (Wie lange hier?) 3 Tage. Heute sei Donnerstag, Oktober 1918. Hat angeblich Ref. niemals gesehen. Ebenso kennt er den Oberarzt nicht, das sei ein Inspektor. Die Farbe der Kreide nennt er falsch. Rot ist blau, schwarz ist weiß, grün ist gelb. $3 \cdot 3=6 ; 4+5=20 ; 2+5=12$. Komme vom Durchgangslager Heilsberg. Sei gefahren Altona-Köln-Berlin. Dann: sei eingekleidet bei den 154 ern in Borowischken, dann 132 ern Straßburg. Kam nach Sibirien in russische Gefangenschaft. War dort 3 Jahre. Floh nach Königsberg. Wußte nicht Bescheid und fuhr weiter nach dem Westen. Hatte 300 Rubel. Seine Uniform war kaput, die Kinder sind ihm nachgelaufen. Das sei richtig, daß er von der Regierung $3 \mathrm{M}$. bekomme für jeden, den er umbringt. Möchte jetzt gern bald weg. Seine Tochter sei in Paderborn bei Frau Meier. Nennt nach 15 Minuten bereits wieder eine andere Adresse.

26. XI. 19. Augenuntersuchung. Kein psychogener Strabismus. Keine Gesichtsfeldeinschränkung.

Beantwortung eines Fragebogens: Gedächtnisleistungen mäßig, Merkfähigkeit sehr mäßig, Kombinationsfähigkeit besonders beim Erkennen von Zusammenhängen auf Bildern schlecht, Urteilsfähigkeit sehr schlecht: Unterschied zwischen Kind und Zwerg? Der Zwerg ist klein und das Kind ist etwas größer. Rechtsanwalt und Staatsanwalt? Rechtsanwalt ist nicht so hoch wie ein Staatsanwalt. Borgen und Schenken? Wenn ich schenke, dann ist das gut, leihen ist auch gut. Geiz und Sparsamkeit? Sparsamkeit ist besser als Geiz. Irrtum und Lüge ? Das ist gar nichts. (An wen zahlt man Steuern?) An die Staatskasse. (Was ist der Zweck der Ehe?) Die Frau. (Wie denken Sie sich Ihre Zukunft?) Gut.

30. XI. 19. In letzter Zeit verhältnismäßig ruhig. Klagt heute über Schlaflosigkeit. Abends sehr heiser. Sagte, er bekäme Anfälle, die hätte er in England gelernt.

1. XII. 19. Jammert und weint nachmittags laut vor sich hin. Auf Zureden ändert or sein Verhalten nicht.

3. XII. 19. Tags und nachts ruhig. Meint, er hätte zuviel Blut verloren und müsse wohl bald sterben. Bei ihm habe das Blut doch gespritzt. Deshalb sei er auch kein Verbrecher. Bei letzteren fließe es nur ganz langsam.

4. XII. 19. Macht weiter sich widersprechende Angaben. Deshalb Elektrosuggestivbehandlung. Behauptet, Wiadrowski zu heißen und in Hamborn i. Westf. zu Hause zu sein. Sei kein Kriegsgefangener, wäre 1916 in Brest-Litowsk desertiert und hätte sich bis 19 herumgetrieben. Ein Verbrechen habe er nicht begangen. Wollte sich die Vorteile eines heimgekehrten Kriegsgefangenen aneignen. Depesche nach Hamborn an die Polizei ohne Erfolg, an die Adresse Wiadrowski zurück mit dem Ergebnis, daß sich jemand gleichen Namens meldet, aber nur auf einem Zufall beruhend. Alles dies macht keinen Eindruck auf P., behauptet jetzt Priedigkeit mit $\mathrm{P}$ zu heißen. Ihm wird gesagt, daß in den nächsten Tagen sein Gehirn freigelegt wird, auf daß er wieder gesund werde. Diese Nachricht macht scheinbar etwas Eindruck. Er wird nachdenklich. Vorbeireden (beim Rechnen und bei Benennung von Gegenständen) vollkommen geschwunden. (Zu Hause?) In Hessen. (Stadt?) Hessen-Nassau. Ist ein Dorf, heißt Hessen-Nassau. (Straße?) Berger Weg. Hessen ist bloß ein Dorf, eine Stadt. (Zu Hause?) Hamm. (Wo liegt das?) Westfalen. (Zu Hause?) Zuletzt bin ich in Barmen gewesen (Zu Hause?) Hamborn, Rhld., geb. in Ostpr. (Zu Hause?) Hamm, Buchersche Heide 48. (Straße?) Nordstr., Beidigstr., Bahnhofstr. (Zu Hause?) Hamm, Bochumer Weg. (Wo erkundigen?) Hamm, Amt, Bürgermeister. (Zu Hause?) Hessen. (Geboren?) Rauno b. Senftenberg, 24. XI. 92. (Wie lange ?) 3--4 Jahre als kleines Kind, dann nach Hessen ge- 
fahren, wohin wisse er nicht mehr. Auf die immer wiederholte Frage, wo er in Hessen zur Schule gegangen, antwortet er stets anders: Kann ihm nicht mehr einfallen; Hessen zuerst zur Sohule gegangen; kann ich doch nicht so wissen; weiß doch nicht ganz genau. Der Lehrer heißt Lehrer Blume. Ich weiß nicht wo. Luisenstraße. Das Dorf heißt Hessen, hegt in Hessen-Nassau. (Dorf ?) Das Dorf heißt . . . murmelt Unverständliches. (Dorf ?) Hessen ist ein Dorf. (Größere Städte in der Nähe?) Hessen-Nassau. Ich bin in Huckegarde in die Schule gegangen. (Wo liegt das?) Westfalen, Rheinland. (Wie lange in die Schule gegangen ?) 6 Jahre, von da nach Merten in Westfalen, von da nach Hamborn, Heinestr. 45 (48) bis 1918. 1915 Soldat geworden in Hamborn bei den 131. 1916 ins Feld gekommen. Zuerst in Bielefeld ausgebildet, dann ins Feld nach Rußland, 1 Jahr hatte etwas die Masurenschlacht mitgemacht. 1915 acht Wochen in Bielefeld ausgebildet, nach Rußland ins Feld zur Bahnbewachung in Brest-Litowsk, dort 2 Jahre, dann nach Frankreich nach der Somme. März 1918 . . . An der Somme bei Mons gefangengenommen 1918 im März. War an der Somme ein halbes Jahr. Im Mai an der Somme gefangen, im Juli in Hiele (?) von den Franzosen. Kam zuerst in eine Stellung, dann verschickt nach einer Irrenanstalt, war im Gefängnis ... . in Belgien, von Löwen . .. im Mai ... zu Frankreich in Nervenanstalt. (Name?) In in ... in Mons in die Nervenanstalt. (Wann nach Deutschland?) Im Juli - Koblenz, bin am 1. Juli dahin gekommen. Wurde am 31. Oktober aus Mons entlassen. Bin am 1. Juli nach Koblenz gekommen, aus Mons weggelaufen, mit 15 Mann durchgebrochen. Über Belgien gegangen, nie angehalten, 2 wurden totgeschossen. Von Mons bis Koblenz 5 Wochen unterwegs. Gelebt von Brot . . . Für 5 Wochen Brot, mitgenommen, 6 Brote. Von den Leuten gekriegt. Macht über Geburtsort, Schule usw. wechselnde Angaben. In die Lehre sei er auf Zeche „Deutscher Kaiser" in Hamborn gegangen. Dort 5 Jahre gewesen. 1915 eingezogen. Ausgebildet in Soest, Bielefeld 131. Hauptmann Schröder. Gibt dann als Name abwechselnd Johann Brüdigkeit, und Franz Wiadrowski an, Vater Georg Wiadrowski, Mutter Luise Pantel usw. Vater war Bergmann auf Zeche „Deutscher Kaiser" in Hamborn, solange er lebte, starb 1915, $50 \mathrm{~J}$. alt. Mutter starb 47 Jahre alt, 1907, Vater war älter. Habe drei Geschwister gehabt; Friedrich, Otto, August, seien im Kriege gefallen, einer in Gefangenschaft. Otto war verheiratet. Frau wohne jetzt noch Heinestr. 48, Hamborn.

Auch weiter dauernd wechselnde Angaben besonders über seine Gefangenschaft. Sohließlich sagte er: Juli 1919 gemeldet in Koblenz, kam ins Lazarett evgel. Stift, dort 3 Wochen. Dann Andernach, dann hierher geschickt, da er gesagt hat, Rauno sei seine Heimat: Kam nach Heilsberg, dort 12-15 Tage, hier seit 10 Tagen. Habe gelogen, weil er in Not war. Jetzt habe er ja was zu essen. Habe hier noch gelogen, weil in den Papieren der Name stünde. (Wirklicher Name?) Franz Wiadrowski. (Krank?) Faßt sich nach dem Kopf. Weint. Schäme sich. Stehe allein in der Stadt. Wisse keinen Freund, keinen Kameraden. Schiele von Kindheit an. Wenig krank, einmal lungenkrank. Habe jetzt das erstemal gelogen. Nie Gefängnis gehabt, wolle ein ordentlicher Mensch werden.

4. XII. 19. P. redet viel, er habe die Engel gesehen. Sie wollten ihn noch nicht haben, er wäre zu gut und zu schön. Schläft nachts wenig.

5. XII. 19. Kommt ins Zimmer mit lachendem Gesicht und fragt in frechem Ton: „Was soll ich denn? Ich habe mir weh getan". Setzt sich, steht zweimal auf. Ist dann ruhig. Ich heiße Priedigkeit Friedrich, geb. in Rauno bei Senftenberg, Kreis Kalau. Eltern leben in Hamm in Westf., Bochumerweg 157. Vater heißt Georg Priedrigkeit, Beruf Bergmann. Mutter heißt Luise Priedigkeit geb. Pantel. (Dienst getan?) - Fahnenflüchtig nicht, keine Strafen, Geld gestohlen $800 \mathrm{Mk}$. 1916 bei der Truppe in Brest-Litowsk, nicht herausgekommen, von der Kriegskasse. 
Ihn hat man nicht gesucht. Weggekommen mit der Truppe. 1 Bagagewagen verkauft 1917 für 10 Rubel, Essen dafür gekauft. Nach Koblenz gereist. Fahnen. flüchtig $3 \mathrm{Jahr}$, gemeldet in Koblenz. 1916-1919 herumgetrieben, blieb bis 1918 in RuBland, geplündert in Mitau. In Deutschland nichts verbrochen. In Koblenz wollte er Papiere holen, kam ins Lazarett, wurde nach Altona geschickt, von da nach Heilsberg. Wäre Kriegsgefangener, wollte Entlassungsanzug haben. Alwin Block habe er umgebracht in Wilna, wollte gegen mich kommen, mit dem Seitengewehr in den Bauch gestoßen. 1917 russischen Leutnant totgemacht in Kowno; er sagte, ich solle zur Seite gehen, da hat er einen Schuß auf mich abgegeben, ich habe ihn erstochen.

5. XII. 19. Redet viel: ,Ihr macht mich noch tot hier." Sein Puls schlage schon wieder fester. Faßt sich dauernd an den Arm. Im Schlaf ängstliches Auf. schreien.

7. XII. 1919. Sang viel, schimpft zwischendurch. Nachts sehr laut. „Ich bin kein Verbrecher, man hat mich in ein Gefängnis gesteckt, aber die sollen noch den Johann Brüdigkeit sehen, was der machen kann."

10. XII. 19. Singt und pfeift. Ißt unregelmäßig. Abends laut, schimpft furchtbar auf die Ärzte. Sagt, er wolle erst ruhig sein, wenn er ein Bad bekomme.

12. XII. 19. Unterhält sich viel mit anderen Patienten; geordnet.

14. XII. 19. Ich heiße Johann Brüdigkeit, wie ich zuerst angegeben habe, habe gar keinen Menschen mehr auf der Welt, stehe ganz allein. Ein Bruder ist in Gefangenschaft, der heißt Otto. Außerdem habe ich noch zwei Schwestern Henriette und Luise, ich weiß nicht, wo die sind, und wo die wohnen. Ich weiß überhaupt nichts, weiß nicht, woher ich gekommen bin. Beide Eltern schon lange tot. - Ruhig.

20. XII. 19. Nachts oft laute Selbstgespräche, will baden und eingepackt werden.

24. XII. 19. Selbstgespräche.

25. XII. 19. Schimpft mittags viel und weint.

28. XII. 19. Ist oft aufgeregt, fängt mit anderen Patienten Streit an.

29. XIL. 19. (Name?) Friedrich Priedrigkeit mit P. (Wo geboren?) Rauno bei Senftenberg, Kreis Calau, Provinz Brandenburg, Prov. Ostpreußen das ist nicht gelogen.

Ich heiße Friedrich Priedrigkeit, geb. am 24. November 1892. Eltern wohnen in Hamm, Bochumerweg 157. In Hamm bin ich 6 Jahre gewesen. 1916 Soldat in Bielefeld, bei den 131., 8 Wochen ausgebildet. Dann nach Haus geschickt, habe gearbeitet auf Zeche Sachsen, in Hessen (?) 1917 bin ich noch einmal Soldat geworden in Bielefeld, Bez.-Kom. Soest. Bin eingezogen, weil ich Bergmann war und tüchtig arbeiten konnte. (Warum eingezogen ?) Weil ich ausgebildet war. (Warum eingezogen ?) Weil ich nicht arbeiten konnte und zu schwach war. I Jahr in Hamm Soldat gewesen. Dann in Anstalt Lengerich, dort 4 Monat, August 1918 nach Haus geschickt. Oktober 1918 von Militär entlassen, dann wieder auf der Zeche gearbeitet. In Hamm auf der Polizei gemeldet. Ich bin kein Kriegsgefangener gewesen. Aus Not habe ich mich in Koblenz gemeldet, weil ich nur $6 \mathrm{Mk}$. verdiente. Ich habe mich gemeldet, weil ich nicht richtig im Kopf war.

loh heiße Friedrich Priedrigkeit, geb. 24. November 1892, Rauno bei Senftenberg, Kreis Calau, Provinz Brandenburg, Provinz Ostpreußen. Meine Elt ern wohnen in Hamm, Bochumer Weg 157. Georg Priedrigkeit, geb. Fischhausen 2 Schwestern, Gertrud, Elise Priedrigkeit, 2 Brüder, Johann und Otto,Priedrigkeit. Eingezogen am 26. Oktober 1917 Bez.-Komm. Soest, von da nach Bielefeld 131, von da nach Halle a. d. Saale. Inf.-Regt. 131, von da nach Moria Bethel, von da nach Lengerich Heilanstalt. 
3l. XII. 19. Bis 11 Uhr sehr laut. Dann auf sein Verlangen gepackt, schrie danach furchtbar. Machte sich um $1 \mathrm{Uhr}$ von der Packung selbst frei. Half sein Bett zurücktragen und schlief dann.

2. I. 20. Brief vom Vater, der bestätigt, daß Pat. sein Sohn ist.

3. I. 20. Schimpft viel. Verlangt dauernd, kalt gebadet zu werden. Gegen Abend laut, viel gesungen.

Anm. Während einer klinischen Vorstellung aufgefordert, Anfälle vorzumachen, tat er dies nach anfänglichem Sträuben (Art des hysterischen Anfalls).

6. I. 20. Fängt an, sinnlose Reden zu führen, er sei der König, sein Königreich sei hier unten. Er hätte hier ermordet werden sollen, zeigt dabei dauernd auf seinen linken Unterarm. Zeitweise sehr laut, wird still, sobald man ihn zur Ruhe ermahnt.

7. I. 20. Schreit: Ich bin der König, ich bin der heilige Christ, schimpft und singt andauernd. Wird am Abend sehr erregt, sprang im Zimmer, ging mit den Fäusten auf andere Patienten. Schrio: Ich bin der König von England. Nach Packung ruhiger.

9.I. 20. Am Tage ruhig, nachts getobt. Versuchte die Schwester anzugreifen, schrie laut: ich bin der König, tanzte im Saal herum; Packung, trotzdem sehr laut. Um 5 Uhr bat er, man solle ihn herausnehmen, er wolle jetzt ruhig sein. Schlief dann auch ein.

1 15. I. 20. Oft sehr verworren in seinen Reden. Macht hin und wieder dieselben Bewegungen, wiegt z. B. den Oberkörper hin und her, klatscht in die Hände, springt aus dem Bett, dann wieder herein. Greift andere Patienten an.

30. I. 20. Beantwortung zweier Fragebogen.

I. (Name?) Kaiser Wihm I. (Beruf ?) kommiz mehr. (Alter?) +. (Wann geboren?) 24. November. (Wo geboren?) Ostpreußen. (Name der Eltern?) Königin Luissi. (Beruf des Vaters?) Hausbesitzter. (Verheiratet?) Königin Tandau. (Wann geheiratet?) 80 nie ich ins Bett. (Wie alt bei der Heirat?) 28 Jahren. (Wieviel Kinder?) 3 Sohne. (Wieviel Kinder gestorben?) ganze Weltkönigin. (Namen der Kinder?) Georg, Otto, Johann. (Alter der Kinder ?) 2 Jahre älter. (Wo zur Schule?) Königin Ereren. (Name der Lehrer?) Bume, Kettler, Olrage. (Name der Mitschüler?) Engschen König. (Wieviel Klassen durchgemacht?) II. (Sitzen geblieben?) I. (Welche Fächer schwer, welche leicht gefallen?) gut. (Welches Jahr?) 37 Wochen 5. (Welchen Monat?) Jahr. (Welches Datum?) Januar. (Welchen Wochentag ?) 7 Woche. (Wielange hier?) 7 Wochen 5. (Wo vor 8 Tagen?) 8 Tagen. legen. (Wo gewohnt?) Hamm. (Einteilung von Jahr, Monat, Tag, Stunde usw. ?) -F. (Wann Pfingsten?) August. (Sommerbeginn?) 25. Juni. (Frühlingsanfang?) 21. März. (Herbstbeginn?) August. (Winterbeginn?) Herbst. (Welche Jahreszeit ?) 1920. (Wo hier (Stadt)?) - . (Welches Haus?) in KönigsschoB. (Wer hat Sie hier her gebracht ?) 2 Soldaten. (Mit was für Leuten im Saale ?) mit Königssohne. (Name des Pflegers?) so wie ... her. (Name der Ärzte?) P. I F. (Krank ?) gesucht. (Traurig?) bezahlen. (Stimmen?) ganze Welt. (Gestalten?) nein. (Verfolgt?) nein.

II. Rechenaufgaben richtig gelöst, nur Dividieren mit größeren Zahlen schlecht. Bei eingekleideten Rechenaufgaben völliges Versagen. Wiederholt nur Zahlen, die in den Aufgaben vorkommen oder multipliziert einfache Zahlen. Einmal lautete die Antwort König.

3. II. 20. (Wie alt?) 28 Jahre. (Haus?) Königshaus. (Datum?) $6 \mathrm{Uhr} 10 \mathrm{Mi}$ nuten genau. (Wieviel Uhr?) Geht verkehrt, meine geht schneller, 9 Uhr 10 Minuten ( $3 / 48 \mathrm{Uhr}$ ) korrigiert sich 9 Minuten vor $10 \mathrm{Uhr}$. (Wie lange hier?) 7 Wochen 8 Tage. (Datum?) Januar 1920. (Anfang, Mitte, Ende?) Ist bald vorbei. (Krank?) Bin gesund. War krank, wurde nichts gemacht. Keine Meldung. (Sie sind doch elek- 
trisiert worden?) ist keine Behandlung für kranke Menschen. (Operiert ?) Niemals. (Elektrisiert?) Ja, so wie ich behandelt, ist kein Mensch. Ich habe mehr getan wie ein anderer auf der Welt. Nach dem Fenster sehend: da muß doch was sein, daß man solche Gitter vortut. Warum tut man das? Weil der Herrscher König ist. (Heilsberg?) Ja wegen Nerven sei er hierher gekommen. (Kriegsgefangener ?) Ich bin ein Herrscher König. Ich sehe doch ganz fremdländisch aus. (Name als König ?) Kaiser Wilhelm I aus Deutschland. Ich bin von der englischen Insel, Baron Rüdesheim, ein Kind. (Rechnen?) Ja. $6+8=14 ; 7 \cdot 9=63 ; 8 \cdot 12=92$; $5 \cdot 7=35 ; 4 \cdot 12=44 ; 3 \cdot 17=$ (wiederholt) $3 \cdot 10=51$. (Wer bin ich [Geheimrat] ?) Gemeinderat.

3. II. 20. Redet dauernd von seinem Königreich.

5. II. 20. Belästigt am Tage die anderen Patienten.

6. II. 20. Schimpft, ruft oft, ich bin der Herrscherkönig, redet dauernd vor sich hin, Selbstgespräche führend.

10. II. 20. Dauernd Schaukelbewegungen. Schimpft oft, redet kindlich wirres Zeug, besonders wenn er sich beobachtet fïhlt. Er ist der König, er ist gut, er darf nicht erschossen werden. Beinahe wäre er getötet. Redet jeden mit Du an.

14. II. 20. Äußert sinnlose Größenideen, ,,er sei der letzte König“, der ,Weltkönig", die anderen Könige stellten ihm nach. Man habe ihn schon viermal ermordet und viermal beerdigt. Zeigt einen Messingring vor und blickt durch denselben nach der Decke; das sei der Beweis, daß er König sei, das könne nur ein König.

3. III. 20. Unverändert. Stets dieselben sinnlosen Größenideen. Begrüßt den Arzt jovial, indem er ihm von weitem mit freundlichem Lächeln die Hand entgegenstreckt, um dann sofort zu beginnen: "Nun habt ihr wieder einen Fehler gemacht, ich habe nun wieder gesiegt. Das dürft ihr doch nicht machen, wenn ihr Ärzte seid. Da siehst du ja. meinen König, meinen Weltkönig, der hat einen dickern Kopf wie deiner. Nun habt ihr ihn ermordet, erschossen habt ihr ihn, und ich habe gesiegt, hier seht Thr ja, daß ich gesiegt habe, das ist mein König, hier (steigt aus dem Bett und deutet auf das Waschbecken), das ist meines und das ist dem Professor seines."

17. III. 20. Wiederholt täglich die gleichen sinnlosen Größenideen und Wendungen fast genau mit den gleichen Worten.

18. III. 20. Assoziationsversuch: Oft einfache Wiederholung des Reizwortes, Neigung zu Ausdrücken wie gut, sehr gut, schön. Viele Reaktionen zeigen die Egozentrizität des Patienten. Auf das Reizwort: verfolgen, erfolgt die Reaktion: "Sie haben mich sehr verfolgt und haben mich doch nicht gekriegt."

31. III. 20. Unverändert, ruhig, läppisch, begrüßt den Arzt stets mit den gleichen Worten: ,jJetzt habe ich euch wieder besiegt, was habt ihr denn da wieder für einen Fehler gemacht? Mein Schreiben seht ihr ja hier, ich habe jetzt die Regierung abgesetzt, mein König hat jetzt gesiegt." Wackelt fast dauernd mit dem Oberkörper hin und her.

20. IV. 20. Im allgemeinen unverändert. Verlangt heute zum erstenmal aus der Klinik heraus, will in die Stadt, Abwechslung. Bringt dabei nach wie vor die gleichen Größenideen vor.

2. V. 20. Auch des Nachts Selbstgespräche.

9. V. 20. Ohne jede Veränderung. Steht auf, beschäftigt sich nicht, sitzt dauernd auf seinem Stuhl, den Oberkörper in automatischer Weise hin und her wiegend. Bringt immer noch die gleichen Ideen vor.

29. V. 20. Unverändert. Läuft im Garten dauernd lebhaft gestikulierend und sprechend hin und her. Bringt immer wieder die gleichen Geschichten vor: er habe gesiegt usw. Spricht auch in der Nacht meist dasselbe. Wird zur Gartenarbeit mit 
anderen Patienten unter Aufsicht eines Pflegers mitgenommen, sucht fortzulaufen, der Pfleger solle selber arbeiten. Mußte auf Station zurückgebracht werden. Hört nur auf den Namen König.

14. V1. 20. Ohne Veränderung. Immer noch lebhaft. Stets dieselben Selbstgespräche.

4. VII. 20. Vom Urlaub zurückgekehrt fragt die Oberschwester P.: ,,Erkennen Sie mich wieder? Wissen Sie, wer ich bin?“, worauf er antwortete: ,Sie sehen ja schlechter aus als Sie vorher ausgesehen haben."

5. VII. 20. Vorstellung im Kolleg: (Name?) ,S-t-u-i-n.“ ,Ich gehöre nach Amerika und nach Italien. Ich bin der Thüringer Hund gewesen. Ich bin hierher gekommen, ich habe 36 Akten gehabt, das sind Militärakten. Aber ich habe es dem Richter gesagt. Ich bin ein gerechter Mensch, ich bin der Donner und der Blitz, ich bin doch mehr als ihr alle seid. Ich werde mir mal erst richtig von China meine Messer kommen lassen und euch den Schädel kaput schlagen. Ihr seid doch alle miteinander dümmer als ich bin." (Wo hier ?) "Ich bin hier in Italien." (Beruf ?) Ich bin von Beruf englische Hoheit.“ (Alter ?) ,104 J. alt bin ich als alter Fritz." Bezeichnet die ihm vorgehaltenen Gegenstände richtig, teils als sein gestohlenes Eigentum: „Das ist meine Uhr. Das ist eine gestohlene Uhrkette, das ist alles gestohlen". Soll seinen Namen an dieTafel schreiben, schreibt: ,S t uin“"

Anm. Gelegentlich wurde P. beim Mühlespielen beobachtet; er spielte korrekt, unterbrach aber das Spiel, sobald jemand hinzukam.

14. VII. 20. Von der Schwester eingeschüchtert tritt P. zögernd und ängstlich ins Untersuchungszimmer. Spricht seine gewohnten Reden sehr laut und lebhaft, setzt sich dabei, läßt sich aber nicht unterbrechen. Läßt sich schließlich beruhigen, wird still. Befolgt alle Aufforderungen richtig. Erneute körperliche Untersuchung. Status idem. Bei der Sensibilitätsprüfung sieht P. sehr ängstlich auf die Nadel, weicht aus, beruhigt sich erst, als er sich überzeugt, daß die Prüfung keine erheblichen Schmerzen bereitet. Bei der Aufforderung, spitz und stumpf anzugeben, wechselt er zunächst automatisch ab: spitz, stumpf, spitz, stumpf. Auf energischen Verhalt gibt er richtigere, aber nicht völlig richtige Antworten. Die ganze Untersuchung verfolgt $P$. mit Spannung. Wieder in Ruhe gelassen, beginnt er - jetzt ohne besonderen Nachdruck - die alten Reden. Ist auf Anfforderung still.

20. VII. 20. Geht im Garten in einem Viereck dauernd herum, führt dabei lebhaft gestikulierend Selbstgespräche. Reagiert prompt auf Anruf mit seinem Namen, kommt heran, beantwortet kurze Fragen richtig, wendet sich aber schnell wieder ab und verfällt in seine alte Beschäftigung. Im Krankensaal stellt sich P. an den Heizkörper und macht Wackelbewegungen. Beschäftigt sich kaum. Nur bisweilen hilft er auf Aufforderung oder spontan Kaffee austeilen, nimmt den Besen zum Reinigen. Meist wirft er ihn bald weg. Hat stets großen Appetit, ißt alles, was andere stehen lassen, sucht beim Austeilen etwas zu erhaschen.

2. VIII. 20. Der Inhalt der Selbstgespräche hat sich geändert. Es werden nicht mehr dieselben Redewendungen und Geschichten vorgebracht. Das Thema wechselt vielmehr von Minute zu Minute. Die Größenideen treten etwas mehr in den Hintergrund. Es wird eine strenge Dialogform eingehalten. Die Themen sind dem Militärleben, dem Leben auf der Zeche, dem Aufenthalt in der hiesigen Anstalt entnommen. Satzbau völlig gewahrt. Keine Neologismen, keine Wortstereotypien. Zwischen den einzelnen Themen besteht eine, wenn auch sehr oberflächliche assoziative Verknüpfung. Bisweilen echolalische Anknüpfung. Meist Anknüpfung an ein Wort, das im Satz nur eine untergeordnete Rolle spielte, seltener ein innerer inhaltlicher Zusammenhang zwischen den Themen. Zwischendurch antwortet P. auf kurze Fragen richtig. Wenn man durch eindringliches Fragen ihn hindert, in seinen Dialog zu verfallen, kann man mit ihm sich fast fünf Minuten 
richtig unterhalten. Das Selbstgespräch knüpft nachher meist an die zuletzt gestellte Frage an. Die Themen sind nicht fixiert, wenn auch eine gewisse Vorliebe für einzelne Themen vorkommt. Wortbetonung normal. Lebhafte Gestikulation. Der Inhalt der Gespräche ist etwa wie folgt:

1. ,Ich war doch Förderaufseher, sagt er. Ich war auf Zeche Devendel. Was hast Du drin gearbeitet, sagt er. Ich bin doch mit der blanken Lampe dagewesen. Hast Du keine Arbeit gemacht? Ich bin doch Aufseher. Ich brauche nicht zu arbeiten. Ich bin doch Hauptmann. Wirst Du denn auch das eintragen? Ich bin doch Hauptmann von Bayern. Stimmt das nicht? ..."“

2. „Ich bin doch Kassenführer der ganzen Staaten. Stimmt das nicht?. Ja. Dann werd' ich auch genug. In Rumänien bin ich doch auch ? Ich bin doch in der ganzen Welt. Ich habe meine Schlachten immer fertig. Die Deutschen sind nicht zu gebrauchen. Wenn ich auf meinem Pferd draufsitze, dann gewinne ich auch. Bin ich nicht immer Kassenführer gewesen? Ja. Ich habe meine drei Schichten immer heraufgebracht ..."

3. „Ich bin beim englischen General. Sonst sind wir nackend gegangen mit so 'nem Ding. Und was ist der eine gewesen? Tiroler. Der wird euch schon wieder falsch gemacht haben. Wir waren doch beide zusammen. Ja. Wie lange haben wir gearbeitet? Der Krup war Schreiber. Und was war Himson? Der war auch Schreiber. Der gehört nach Berlin. Ich gehöre nach Berlin. Das habt ihr wieder falsch gemach, ..."

4. „Kennst du die Leute von der Insel ? Die Insel ist doch gefroren. Ich neulich weg. Warum gehst du weg? Ich muß doch die Verbrecher rauskriegen. Die Insel weiß Bescheid. Sind die Leute nicht hier gewesen? Ja, stimmt doch. Bist du vorher nicht in Hamm gewesen? Ja, stimmt. Hast du die Kohlen gefahren ? Ja ..."

5. „Hast du den Kataschka gekannt? Warum warst du bei Kataschka? Der belgische König heißt immer so. Heißt so von Kind an. Hast du Kataschka gesehen? Den bayrischen Idiot hast du gesehen. Ich werde dich rühmen, sagt Norsche. Du bist ein guter Mann. Wirst du nicht reinfallen? Sind alle reingefallen, jetzt, sagt der Hauptmann. Kennst du mein Geld, was du gekriegt hast? Nein, keinen Pfennig! Lassen wir es liegen, verfaulen. Ja werde ich es auch bekommen? Kennst du den Hauptmann von Bayern? Ja, ich habe das Geld ..."

6. „Das brauch' ich nicht zu wissen. Wissen meine Eltern allein. Oder brauchen sie nicht zu wissen, sagt Norsche. Die Norsche war bei den Eltern, war beim englischen General. Ist sie dagewesen, sagt er. Ja. Trachten sie ihr nach dem Leben? Ja, weil sie nicht arbeiten. Das hat der englische rausgekriegt. Was haben sie denn getan? Sie haben mich elektrisiert und geschnitten am Kopf. Ja. Werd' ich sagen, daß sie das nicht dürfen. Weiß das dein Bruder, daß du immer ein Verbrecher warst?..."

Es wird $P$. in Aussicht gestellt, $\mathrm{da} ß$ er nichts vom Gericht zu befürchten habe und daß er bei geordnetem Verhalten auf die ruhige Abteilung verlegt und bald nach Hause entlassen werde.

6. VIII. 20. Weit zugänglicher, man kann fünf Minuten mit ihm vernünftig reden. Als man ihm sagt: ,Na nun werden Sie bald von hier loskommen, Priedrigkeit!" antwortet er: „Ja, in 14 Tagen, nicht wahr ?" Verfällt dann wieder in seine Selbstgespräche. Beim Versuch, ihn zu reizen, steht er erregt auf, nimmt drohende Haltung ein, geht aber nicht weiter, spricht: ,du bist verrückt, ich bin gescheiter wie du, ich werde meine englischen Heere holen usw." Schreit:, du hast mir gar nichts zu sagen! Ihr könnt mir gar nichts machen." Läßt sich bald beruhigen. Wackelt übrigens heute bei der Unterhaltung nicht.

10. VIII. 20. Auf die Nervenstation verlegt, dort unruhig, durch seine Gespräche störend, muß wieder zurückverlegt werden. 
15. VIII. 20. Wird zum Gottesdienst zum Hörsaal mitgenommen. Blätterte in seinem Gesangbuche, las etwas, dichtete sich etwas hinzu und sang dabei immer die gleiche Soldatenmelodie. Als alles still wurde und aufstand, stand er mit auf. War etwa 2 Minuten ruhig. Dann drehte er plötzlich den Kopf zur Seite, sprach zunächst vom Pfarrer, dann von England usw. Wurde so laut, da $B$ er herausgeführt werden mußte.

30. VIII. 20. Augenuntersuchung. Selbstgespräche, benimmt sich aber sonst ruhig, so daß er die anderen Patienten nicht stört. Erste Untersuchung ohne Schwierigkeiten. Pupillenunruhe -+ . Zum 2. Male aufgefordert, sich an den Apparat zu setzen, sagt er, erstaunt aufblickend: ,Was, ich bin doch schon mal vorhin untersucht." Hält dann nicht still, beginnt: „Was wollt ihr denn wieder aus meinen Augen schen, ihr könnt hineinsehen, soviel ihr wollt usw."

7. IX. 20. Inmmer dasselbe einförmige Bild, geht im Garten in einem Viereck herum, führt ständig Selbstgespräche, z. T. auch des Nachts. Im Zimmer stellt er sich an den Heizkörper und wackelt. Aufforderungen führt er richtig aus, geht aber bald wieder in den Garten. Keine Neigung, sich irgendwie spontan zu be. schäftigen. Benehmen sonst geordnet, nicht manieriert. Von den anderen Patienten nimmt er keine Notiz. Stimmung zufrieden. Keine Interessen, keine Sorgen um die Zukunft. Äußert nie etwas von Halluzinationen. Außer den Größenideen sind in seinen Gesprächen keine Wahnideen enthalten. Spricht jetzt viel von „Agaria Bulgarca Kascha“. Beim Nachstenographieren seiner Reden bemerkt P. den Zettel mit den Fragen, die ihm vorgelegt wurden, und sagt neugierig hinsehend und lächelnd: „Ach, das habt ihr ja alles auf dem Zettel aufgeschrieben. Das müßt ihr euch auch noch alles aufschreiben. Ja, ja, das seh" ich wohl usw." Abderhalden (Gehirn, Testikel) -.

Zusammenfassung: Patient erblich nicht belastet. In der Vorgeschichte keine degenerativen, psychopathischen Züge, nichts Schizophrenes. Auf der Schule schwer gelernt, Kenntnisse mangelhaft. Nach der Schule meist auf der Zeche gearbeitet. In keiner Weise auffällig. 1916 eingezogen, auf Reklamation entlassen. 1917 zum 2 ten Male eingezogen. Angaben über sein Verhalten beim Militär nicht zu erlangen. Kam aus unbekannten Gründen nach Bethel, von dort nach Le ngerich (Diagnose: angeborener Schwachsinn). Am 20. X. 1918 als dienstunbrauchbar entlassen. Bis März 1919 auf der Zeche gearbeitet. Über die Zeit vom März bis 1. November 1919 nichts bekannt. Am 1. XI. 1919 erschien Patient im Durchgangslager Heilsberg und gab sich als heimkehrender Kriegsgefangener aus. Da er sich in Widersprüche verwickelte, schöpfte man Verdacht, daß seine Angaben nicht stimmten, daß er sich die Vorteile eines heimkehrenden Kriegsgefangenen aneignen wolle. Beim Vernehmen machte er den Eindruck eines geistig nicht gesunden Menschen, weshalb er der hiesigen Klinik überwiesen wurde. Hier bot er zunächst das Bild eines Gan erkranken mit Vorbeireden und dauernd wechselnden Angaben über Person und angebliche Kriegsgefangenschaft. Auf Elektrosuggestivbehandlung fast völliges Weichen der Krankheitserscheinungen. Ende Dezember 1919 erneut psychotische Störungen, diesmal von anderer Färbung. Größenideen, einförmiges sinnloses Reden, stereotype Bewegungen. Verhalten allmählich ab- 
lehnender, weniger reaktiv, sonst aber geordnet. Ende Juli 1920 Zurücktreten der Größenideen; fast dauernde Selbstgespräche mit scheinbarer Inkohärenz des Gedankenganges sowie Stereotypien. Verlust der Spontaneität, ablehnendes, autistisches Verhalten.

Es handelt sich also um einen imbezillen Menschen, der in der Schule schlecht gelernt hat, im Leben aber zunächst weiter gekommen zu sein scheint, von dem wir schließlich für kürzere Zeit die Spur verlieren, bis er endlich als Geisteskranker wieder auftaucht. Es ergibt sich die Frage: Ist Patient geisteskrank im engeren Sinne - wir müßten dann an eine Dementia praecox denken - oder liegt eine rein psychogene Psychose vor? Sicher ist, daß Patient imbezill ist. Das beweist die Vorgeschichte, die Beobachtung in Lengerich und das hiesige Untersuchungsergebnis.

Das Rechnen, besonders das Dividieren ist sehr mangelhaft, die geographischen, politischen und sonstigen Kenntnisse sind völlig ungenügend, etwas besser sind seine Religionskenntnisse. Seine Kombinationsfähigkeit besonders beim Erkennen von Zusammenhängen auf Bildern, noch mehr seine Urteilsfähigkeit ist schlecht. Beim Assoziationsversuch ist die Reaktionszeit verlängert; das öftere Wiederholen des Reizwortes deutet auf Ärmlichkeit der Vorstellungen hin, das Antworten in Sätzen zeigt mangelhaftes Abstraktionsvermögen und Schuleinstellung; ferner finden sich viele Eigenbeziehungen sowie affektbezeichnende Reaktionsworte und kindliche Werturteile. Die Schrift des P. ist unbeholfen, strotzt von othographischen Fehlern, so daß bei manchen Worten der Sinn kaum zu erraten ist.

Auf dem Boden dieser Imbezillität also entwickelt sich eine Psychose, die anfangs nur als rein psychogene aufgefaßt werden konnte. Für das Zustandekommen dieser Psychose scheint die Zeit vom März bis November 1919 von Bedeutung zu sein, über die etwas Sicheres nicht bekannt ist. Vielleicht ist Patient nach dem Verlassen des elterlichen Wohnorts in Not geraten. Seine Bemerkung vom 25. XI. 1919, seine Uniform sei kaput gewesen, die Kinder seien ihm nachgelaufen, könnte darauf bezogen werden, auch die Angabe vom 29. XII. 1919 (an welchem Tage alle kontrollierbaren Äußerungen den Tatsachen entsprechen): , .... ich bin kein Kriegsgefangener gewesen, aus Not habe ich mich in Koblenz gemeldet, weil ich nur $6 \mathrm{Mk}$. verdiente usw. "Wahrscheinlich hat sich Patient schon in Koblenz als Kriegsgefangener ausgegeben, kam dort zunächst in ein Lazarett, dann nach Andernach und wurde schließlich dem Durchgangslager Heilsberg überwiesen. Auch manche andern Aussagen des Patienten deuten darauf hin, daß er zu dieser Zeit ein Gefühl der Insuffizzenz und Hilfslosigkeit gehabt hat. ,,4. XII. 1919 ... hier habe er gelogen, weil er in Not war, jetzt habe er ja was zu essen usw. ..." Seine Absicht am 1. XI. 1919 in Heilsberg war offenbar, vorläufig untergebracht zu sein, möglicherweise auch sich einen Entlassungsanzug sowie das Entlassungsgeld eines heimgekehrten Kriegs. gefangenen zu erschwindeln (eigene Aussage vom 4. XII. 1919). Aufgefallen durch sich widersprechende Äußerungen erklärte er, er wolle 
alle totschlagen, er bekäme von der Regierung für jeden $3 \mathrm{Mk}$. Diese Äußerung ist vielleicht weniger Ausfluß einer Wahnidee als vielmehr ein mehr oder weniger bewußter bzw. unbewußter kindlicher Versuch, geisteskrank zu erscheinen. War schon in 2 oder 3 Anstalten gewesen, kannte sich daher aus und nahm, als man in Heilsberg ihn des Betrugs verdächtigte, seine Zuflucht zur Psychose.

In der Klinik bot Patient zunächst das Bild eines Ganserkranken :

Ausgesprochenes Vorbeireden beim Rechnen, bei Bezeichnung von Gegenständen, bei seinen Personalangaben usw. Eingehen auf den Sinn der Fragen, die er also richtig versteht. Wenn auch die in der Krankengeschichte eingeführten Beispiele nicht ausreichend sind, um wie Pic $\mathrm{k}$ das Vorbeireden genauer zu charakterisieren, so kann doch nach dem sonstigen Befund kaum bezweifelt werden, daß es sich um typische Ganserantworten handelt, wenn auch zugegeben werden muß, daß das negativistische Vorbeireden dem hysterischen Vorbeireden ähneln und den Eindruck einer Simulation machen kann. (Vgl. das von Kraepelin in seinem Lehrbuch, 8. Aufl., S. 689, angeführte Beispiel.)

Vieles erweckt geradezu den Verdacht einer Simulation. Patient gibt seinen Namen falsch an, nennt sich Brüdigkeit statt Priedigkeit, legt sich den Vornamen eines seiner Brüder bei, macht dauernd sich widersprechende Angaben hinsichtlich seiner Person, seiner Gefangenschaft usw. Er nennt ein falsches Datum, weiß aber, daß am nächsten Montag sein Geburtstag ist. Die Personal-. und Ortsangaben sind nicht einfach frei erfunden, sondern es handelt sich um Vertauschungen, die manchmal kaum zu entwirren sind. Manche scheinbar sinnlose Antwort ist keineswegs so sinnlos, wie es zunächst scheint, sondern läßt sich einleuchtend erklären. So gibt z. B. im ersten Fragebogen vom 17. XI. 1919 Patient sein Alter auf 27 Jahre an, das seiner Kinder auf 28, 29 Jahre. Dies erklärt sich so: Auf die Frage, wieviel Kinder er habe, antwortete er 5. Statt der Zahl der Kinder nennt er die Zahl seiner Geschwister, er hat nämlich 3 ältere Brüder, 2 jüngere Schwestern, Auf die folgende Frage nach dem Namen seiner Kinder gibt er im Sinne des obenerwähnten Vorbeiredens die Namen seiner Geschwister an: Friedrich, Georg, Otto, II. Mädchen. Und die nächste Antwort $(28,29)$ gibt konsequenterweise das Alter seiner ältern Brüder an. Örtlich und zeitlich ist Patient orientiert. Er weiß genau, wann sein Geburtstag ist, wieviel Tage er sich in der Klinik befindet usw. Eine stärkere Bewußtseinstrübung läßt sich nicht nachweisen. Die Beziehungen des Patienten zur Umgebung sind ebenfalls nicht sonderlich gestört. Die Stimmung ist in der nächsten Zeit verzweifelt, ängstlich. Patient schlägt sich vor den Kopf, fragt, warum er nicht loskäme; später sagt er, er wolle etwas anstellen, wenn er nicht entlassen werde. Der Druck des Ungewissen, was mit ihm nun geschehen solle, die Angst vor der Strafe, die Furcht festgehalten zu werden, lastet auf ihm. Am 3. XII. 1919 äußert er die depressive Idee, er habe zuviel Blut verloren und müsse bald sterben, 
er sei kein Verbrecher. Zwei Tage später sagt er, man macht ihn hier noch tot, schreit im Schlaf ängstlich auf. Halluzinationen bestehen nicht. Wahnideen im Sinne der Beziehung, der Beeinträchtigung und Verfolgung spielen keine Rolle. In seinen Schriftstücken zeigt Patient einen eigenartigen Telegrammstil, der zuweilen etwas an die kindliche Sprache erinnert: ,.... Ich wieder verzogen nach Hinterte. I Jahr. Und dann ich nach Rahm im Schmidhorst... das erst arbeit Hamborn usw." Überhaupt zeigt das Benehmen des Patienten puerilistische Züge. Manchmal ist Patient unruhig, laut, schreit, verlangt, man solle ihn einpacken, er wolle dann ruhig sein. Ein anderes Mal, wenn er gepackt ist, verlangt er, frei gemacht zu werden, dann wolle er ruhig sein und schlafen. Es erinnert dies an kindliche Laune und Eigensinn. Vielleicht könnte man auch das Führen von Selbstgesprächen das jetzt bereits in Erscheinung tritt, sowie das später auftretende Duzen als puerilistische Züge auffassen. Patient ist beeinflußbar, suggestibel, läßt sich leicht beruhigen oder wird laut, wenn andere Patienten laut werden. Die Erfahrungen von früheren Anstaltsaufenthalten werden verwertet. Patient verlangt Packungen und Beruhigungsmittel, sagt, er werde jetzt Anfälle bekommen, die er in England gelernt habe. In einer klinischen Vorstellung macht er nach anfänglichem Sträuben einen hysterieformen Anfall. vor.

Am 4. XII. 1919 wurde eine Elektrosuggestivbehandlung mit Starkstrom versucht. Außerdem wurde gesagt, daß sein Gehirn in den nächsten Tagen freigelegt werde. Der Erfolg war eklatant. Das Vorbeireden beim Rechnen und bei Benennung von Gegenständen hörte auf, die Angaben über seine Person, seine Gefangenschaft waren zunächst noch falsch, besserten sich aber zusehends. Am nächsten Tage stimmen seine Personalangaben eigentlich völlig, nur hinsichtlich der Gefangenschaft sind seine Angaben noch nicht zutreffend. Aber auch das schwindet. Patient gibt jetzt zu, daß er gelogen habe, er sei kein Kriegsgefangener, er habe aus Not gehandelt. Zugleich wird er depressiv verstimmt, schreit des Nachts bisweilen ängstlich auf. Er berichtet über eine Art hysterischer Halluzination, sieht die Engel im Himmel, die ihn noch nicht haben wollen, da er zu gut und schön sei. Am nächsten Tage äußert er: man mache ihn hier noch tot, sein Puls schlüge schon wieder fester. Am 29. XII. 1919 bot Patient keine besonderen Krankheitserscheinungen mehr. Übrigens hat Patient möglicherweise den Namen Wia. droswki, den er vor dem Elektrisieren sich nur ein einziges Mal zugelegt haben soll, der nach demselben aber eine große Rolle spielt, nur ange. geben, weil man ihm nicht glaubte, daß er Priedrigkeit heiße. Das Verhalten des Patienten während der ersten Psychose ist wie auch späteı stets geordnet. Die Beziehung zur Umgebung ist erhalten. Patient unterhält sich mit andern Patienten, sucht sie zu beeinflussen, zank1 
sich mit ihnen, hetzt sie auf. Er ist reizbar, wird aber nie aggressiv, weiß stets, wie weit er zu gehen hat. Er haßt den Arzt, der das Elektrisieren angeordnet hat. Von affektiver Verblödung kann nicht die Rede sein. Der ganzen Psychose scheint der Affekt der Angst zugrunde zu liegen.

Angesichts des Zustandsbildes des Kranken bei der Aufnahme und zu Beginn des Aufenthalts in der Klinik kommt eine andere Diagnose als Ganserscher Dämmerzustand bzw. psychogene Erkrankung kaum in Betracht. Die Entstehung nach psychischem Trauma, das Gansersche Vorbeireden, die Ähnlichkeit mit Simulation, das reaktive Verhalten des Patienten die Suggestibilität, das Interesse für die Umgebung, das puerillistische, kindlich eigensinnige Benehmen, das geschickte Ausnutzen von Kenntnissen aus dem früheren Anstaltsleben, die prompte Wirkung der Elektrosuggestivtherapie passen treffend zu dem Bilde. Für Dementia praecox lag damals kein Anhaltspunkt vor. Im Laufe der Beobachtung aber verlor sich das Vorbeireden. Es treten jetzt Größenideen auf, Patient führt einförmige, sinnlose Reden, es bilden sich mit der Zeit stereotype Bewegungen aus, der Gedankengang wird inkohärenter, die Antworten sind bisweilen beziehungslos. Ende Juli treten die Größenideen in den Hintergrund. Patient führt jetzt ständig Selbstgespräche mit Inkohärenz des Gedankenganges, geht dauernd im Garten im Kreise herum oder macht stereotype Bewegungen, wird ablehnend, beschäftigt sich nicht. Der Zustand wird immer weniger beeinflußbar, Patient ist völlig affektlos, hat keine Klagen und Wünsche, kein Interesse für die Umgebung, ist autistisch. Betrachtet man das Krankheitsbild nach mehrmonatigem Verlauf oder gar in seiner jetzigen Ausbildung ohne die Vorgeschichte und Entwickelung, so müßte man eine Dementia praecox annehmen. Die Beobachtung des Längsschnitts sowie die psychologische Zergliederung der als schizophrene Symptome imponierenden, Erscheinungen zeigt aber, daß auch eine andere Deutung möglich ist.

Nach einer kurzen, von Krankheitserscheinungen fast völlig freien Zeit entwickelt sich nach dem Ganserschen Dämmerzustand etwa Ende Dezember 1919 eine neue Psychose. Ihr Zustandekommen könnte man sich vielleicht folgendermaßen erklären: Patient ist fast völlig normal. Seinen jetzt wirklich richtigen Angaben wird nicht geglaubt (weil sie z. Zt. unkontrollierbar waren). Er sieht, daß er auch so nicht weiterkommt. Er wird weiter festgehalten. Er steht unter einem gewissen psychischen Druck. Er sieht, daß, wenn er jetzt gesund wird und entlassen werden könnte, wieder die Gefahr der Bestrafung näherrückt, daß die Not, der er ja entgehen wollte, wieder an ihn herantritt. Der Aufenthalt in der Anstalt ist für ihn das bessere Los. Die Ursachen sind vielleicht geeignet für ein abermaliges Zustandekommen einer Psychose, die nun eine andere Färbung aufweist. 
Patient beginnt jetzt Größenideen zu äußern, er sei König, Kaiser, Kaiser Wilhelm von Deutschland, dann er sei der alte Fritz, seine Frau sei die Königin Luise, er befinde sich in einem Königschlosse, „da muß doch was sein, daß man solche Gitter vortut. Warum tut man das ? Weil der Herrscher König ist". Die Patienten sind Königssöhne. Später : er sei bayrischer Hauptmann usw. Die Namen und Bezeichnungen, die sich Patient zulegt, scheinen nicht einfach frei erfunden, vielmehr von bekannten Personen und Dingen, entlehnt. Da nennt sich Patient z. B. ,alter Fritz" (er heißt Friedrich), dann ,deutscher Kaiser", „Kaiser Wilhelm von Deutschland“ (er hat auf der Zeche Deutscher Kaiser gearbeitet), seine Frau sei die Königin Luise (seine Mutter heißt Luise), er sei bayrischer Hauptmann, Hauptmann von Bayern (sein Kompagnieführer, der ihn ausbildete, war Hauptmann, er spielt auch in den späteren Gesprächen eine große Rolle) usw. Ferner führt Patient viel Gespräche mit sich selbst und anderen. Sein Wahn wie sein sonstiges Reden zeigt Konsequenz, Methode. Assoziative Verknüpfung auch an vorausgegangene Erlebnisse ist überall vorhanden. Es besteht keine Inkohärenz bei näherem Zusehen. Es ist nicht das Unverständliche, Verblüffende, das Zerfahrene, Befremdende, psychologisch Unverständliche eines schizophrenen Wahns. Die Äußerungen des Patienten sind derart, als ob er einen Kampf führt gegen seine Widersacher, die Ärzte, die ihn hier festhalten, wie einen Verbrecher, und die Richter, die ihn beschuldigt haben und bestrafen wollen. Die Ärzte behandeln ihn falsch, halten ihn zurück. Er ist aber nicht so dumm, er wird ihnen schon zeigen, was er kann. Sie können ihm ja doch nichts anhaben, er wird sie zuletzt doch noch alle auslachen: ,Nun habt ihr wieder einen Fehler gemacht, ich habe nun wieder gesiegt, das dürft ihr doch nicht machen, wenn ihr Ärzte seid, da siehst du ja meinen König, meinen. Weltkönig, der hat einen dickern Kopf wie deiner, nun habt ihr ihn ermordet, erschossen habt ihr ihn, und ich habe gesiegt, hier seht ihr ja, daß ich gesiegt habe." Wir sehen bei dem Wahn eine geschichtliche Entwickelung und fortschreitende Verknüpfung. Bisweilen kommt noch vereinzeltes Vorbeireden vor. Das Benehmen des Patienten ist kindlich, albern, läppisch, bisweilen spielerisch.

An auffälligen Erscheinungen haben wir ferner noch die Wiegebewegungen. Sie sind, ebensowenig wie die Redewendungen, stereotyp fixiert. Sie werden nur eine gewisse Zeit hindurch festgehalten, treten zeitweise ganz in den Hintergrund. Im übrigen ist das Verhalten des Patienten weniger reaktiv als früher. Verlegung auf eine andere Station, Aussicht auf Entlassung, Zusicherung von Straffreiheit bleiben ohne Wirkung. Auch ist Patient jetzt ablehnender, beschäftigt sich nicht, ist interessenlos. Alles dies mit Unterbrechungen. So verrät Beobachtung und Interesse für die Umgebung eine Bemerkung des Patienten der 
Oberschwester' gegenüber, die vom Urlaub zurückgekehrt war: „Sie sehen ja schlechter aus, als Sie vorher ausgesehen haben." Bisweilen spielt Patient unbeobachtet Mühle, hört auf, wenn er beobachtet wird. Am 20. IV. 1920 will Patient aus der Klinik, in die Stadt, Abwechselung. Am 29. V. 1920 sucht er bei der Arbeit zu entweichen. Das Verhalten ist im übrigen geordnet. Keine Verschrobenheiten, keine Absurditäten, keine Unverständlichkeiten. Stimmung kindlich vergnügt. Weder spontan noch auf Befragen äußert Patient etwas über die Halluzinationen. Außer den Größenideen keine Beziehungs-, Verfolgungs- oder andere Wahnideen. Die Erregung hat nichts Katatonisches. Es sind keine sinnlosen, läppischen, stereotypen Bewegungen, die Erregung hat immer eine affektive Grundlage. Patient ist reizbar, schimpft, droht mit Worten evtl. auch mit seiner Haltung, geht aber nie zu Tätlichkeiten über.

In den Fragebogen vom 30. I. 1920 kommen zunächst die Größenideen zum Ausdruck. Leicht angedeutet ist hier auch noch das Vorbeireden. Die Fragen nach Anzahl, Name und Alter der Kinder werden in ähnlicher Weise wie früher beantwortet. Auch bei falschen Antworten besteht meist noch eine Beziehung zur Frage: Sind Sie verheiratet? „So nie ich ins Bett"“; bei allen freilich nicht mehr. Rechnen verhältnismäBig gut, wenn auch etwas schlechter als früher. Im Juli ändert sich der Zustand des. Patienten. In der letzten klinischen Vorstellung gibt Patient den unverständlichen Namen Stuin an. Bei der körperlichen Untersuchung am 14. VII.' 1920 erweist er sich als suggestibel. Er läßt sich Angst suggerieren. Bei der Aufforderung, spitz und stumpf zu unterscheiden, wählt er zunächst in kindlicher Weise die einfachste Art, indem er, regelmäßig abwecheslnd, sagt: spitz, stumpf. Auf energisches Anfahren werden die Antworten richtiger. Gegen Ende des Monats nehmen die Selbstgespräche einen andern Inhalt an. Es handelt sich nicht mehr um die ständig wiederholten Redensarten, um den methodischen Wahn, sondern um Gespräche, bei denen eine strenge Dialogform gewahrt wird. Das Thema wechselt von Minute zu Minute. Auch bei der systematischen Forschung nach schizophrenen, Sprachstörungen finden sich keine Wortstereotypien, kein Verbigerieren, keine Wortspielereien, keine Wortneubildungen bis auf die noch zu erwähnenden, keineWortverdrehungen, keine sinnlosen Reimereien. Der sprachliche Ausdruck ist ohne Besonderheiten. Hebung und Senkung ist vorhanden. Keine abnorme Betonung, keine gezierte Sprache. Nichts von Störungen der innern Sprache. Keine Haften bleiben an demselben Gedankengang. Satzbau völlig gewahrt. Bisweilen findet sich ein Abgleiten von einem Vorstellungsgebiet in ein anderes, aber es lassen sich auch da noch gewisse Beziehungen erkennen. Von einer Verflachung des Inhalts kann man nicht sprechen. Natürlich ist der Inhalt der Dialogszenen einfach, naiv, der ganzen sonstigen Denkweise des Patienten entsprechend, aber so 
oberflächlich wie bei Schizophrenen, bei denen man einen Inhalt manchmal überhaupt nicht angeben kann, ist er nicht. Rededrang scheint nicht zu bestehen. Auf Fragen und Anruf hält Patient augenblicklich inne. Kurze Fragen beantwortet er richtig und sinngemäß. Meist verfällt er aber bald in seine Dialoge, zunächst an den Inhalt der letzten Frage anknüpfend. Die einzelnen Sätze sind richtig assoziativ miteinander verknüpft. Die einzelnen Themen dagegen sind nur äußerst oberflächlich miteinander assoziativ verbunden. Bisweilen wird echolalisch angeknüpft. z. B. ,... Sagte der Hauptmann...", hält einen Augenblick inne, dann, ,bin ich kein guter Ma n n, ich bin doch ein guter Mann, sagt Norsche..." Kommt dann auf ein anderes Thema, meist Anknüpfung an ein Wort im letzten Satz, das eine nebensächliche Bedeutung hatte, nur bisweilen besteht ein mehr innerlicher, inhaltlicher Zusammenhang. Die Themen sind nicht stereotyp fixiert, im Gegenteil, sie schließen sich inhaltlich an Fragen und Vorgänge der Umgebung an. Bei der Augenuntersuchung sprach Patient, als er ungeduldig wurde, zunächst von der augenärztlichen Untersuchung usw. Von verworrener, zusammenhangloser, zerfahrener Sprache ist jedenfalls keine Rede. Wohl kommen vereinzelt in seinen Redereien Sätze vor, die uns nicht verständlich sind: "... die Insel ist doch gefroren“, ,sonst sind wir nackend gegangen mit so'nem Ding". Aber jedenfalls sind solche Äußerungen eminent selten. Nun zu den Wortneubildungen: „Himson, Norsche“, Agaria Bulgarca Kastha“. Diese Worte erinnern an polnische und russische Namen, bedeuten vielleicht Anklänge an die Tätigkeit des Patienten in Rheinland und Westfalen, wo polnische Elemente auf den Gruben vertreten sind, wurden vielleicht später verstärkt, als Patient nach eigenen Angaben, Kriegsgefangene bewacht hat. Dafür spricht, daß es nur Personennamen sind, die eigentümlich benannt werden; immer besteht eine Beziehung zu seinen Größenideen. Agaria Bulgarca Kascha ist der belgische König, er sei der belgische König usw. Vielleicht handelt es sich mehr um kindliche Wortspielereien als um wirkliche Wortneubildungen im Sinne der Dementia praecox.

Ich wende mich jetzt zur Frage der Stereotypien. Da kommt das Hin- und Herwiegen des Oberkörpers in Betracht, das jetzt mehr als früher in den Vordergrund tritt. Hier ist zu bemerken, daß Patient sonst geordnet, nicht manieriert ist. Auch geschieht das Wackeln nicht unablässig. Manchmal fehlt es stundenlang. Patient wackelt im allgemeinen dann, wenn er unbeschäftigt im Zimmer sitzt. Bei Regenwetter steht er an der Heizung und wackelt unablässig den ganzen Tag. Draußen geht er in einem Viereck herum, Selbstgespräche führend. Auch das kann als Stereotypie aufgefaßt werden, ist aber möglicherweise auch Ausdruck seiner Imbezillität. Patient ist viel ablehnender geworden, ist weit weniger suggestibel, ist viel weniger reaktiv. Der Vorschlag 
einer Verlegung auf eine andere Station, das Vorhalten, daß er bald entlassen werde, daß er keine Strafe zu erwarten habe, macht keinen nachhaltigen Eindruck auf ihn. Wir könnten eine Erklärung darin finden, daß Patient sich geborgen, jeder Not enthoben weiß und als Imbeziller nicht das Bedürfnis hat, wieder unter die Menschen zu gehen, sich selbständig zu beschäftigen. In der Kirche übt der neue Eindruck eine sichtliche Wirkung auf ihn aus, aber sehr bald verfällt er wieder in sein altes Verhalten. Es ist auch schwieriger als früher, ihn aus seinen Dialogen herauszubringen und kurze Zeit zum vernünftigen Antworten zu bewegen. Die Vorgänge der Umgebung beobachtet er richtig, bisweilen nicht ganz ohne Interesse, macht sogar zutreffende Bemerkungen. Patient beschäftigt sich auch nicht spontan, hat keine Sorgen; kümmert sich nicht um die Umgebung. Seine Affektlage ist gleichgültig. Trotz des ablehnenden, wenig suggestiblen, wenig reaktiven, autistischen Wesens ist sein Benehmen sonst geordnet. Es fehlt jede Verschrobenheit. Er hat keine Manieren, kein läppisches Lachen, keine spontanen Triebhandlungen, kein Gesichterschneiden, überhaupt sonst keine katatonen Symptome, keine plötzlichen unmotivierten Erregungen, kein läppisches Benehmen. Die Stimmung ist kindlich vergnügt, zufrieden. Patient kann gereizt werden, ist leicht zu beruhigen. Andeutungen von Halluzinationen, Beziehungsideen, sonstige Wahnideen fehlen abgesehen von den Größenideen, die jetzt immer mehr in den Hintergrund treten.

Es sei noch des körperlichen Befundes gedacht. Die Herabsetzung der Schmerzempfindung ist eine bei Degenerierten nicht selten vorkommende Erscheinung, freilich auch bei Dementia praecox beobachtet. Das Vorhandensein der Pupillenunruhe ist vielleicht gegen Dementia praecox verwertbar - mit Vorsicht natürlich. Die negative Abderhaldensche serologische Blutreaktion ist der Fehlerquellen wegen nicht ausschlaggebend, kann aber immerhin unsern Zweifel an der Diagnose Dementia praecox verstärken.

Wir kommen mithin zu dem Schluß, daß das Zustandsbild des Kranken, zu Beginn den Eindruck einer rein psychogenen, Erkrankung machte, daß die später hinzukommenden als schizophren imponierenden Erscheinungen, auf die im Zweifelsfalle allein Gewicht zu legen ist, bei psychologischer Zergliederung auch als psychogen aufgefaßt werden können. Ein sicheres Urteil läßt sich aber nicht fällen. Es ist durchaus möglich, daß die gegenwärtig fragliche bzw. nur scheinbare Inkohärenz einer wirklichen Inkohärenz Platz macht, daß das Verhalten des Patienten, bei dem man heute schwanken kann, ob es motiviert (reaktiv) oder unmotiviert ist, allmählich zweifellos unmotiviert wird, daß es sich also nicht mehr um eine psychogene Erkrankung handelt, sondern, daß die Summe der Erscheinungen Ausdruck der Spaltung der Persönlichkeit ist. 


\section{Literatur.}

1) Abraham, Die psychosexuellen Differenzen der Hysterie und der Dementia praecox. Zentralbl. f. Nervenh. u. Psych. 1908, S. 521. - $\left.{ }^{2}\right)$ Aschaffen burg, Degen. Psychosen und Dem. praecox bei Kriminellen. Zeitschr. f. d. ges. Neurol. u. Psychiatr., Orig., 14. 1913. - ${ }^{3}$ ) Austregesilo, L'hystérie et le syndrome hystéroide. Bull. de la Société de Médecine mentale belgique 1913, S. 434. - 4) Baccelli (Brescia), Tic aerofagio e Demenza precoce. Riv. ital. di neuropatol., psichiatr. ed elettroterap. 11, H. 7. - ${ }^{5}$ ) Becker, W., H., Zur Diagnose der Dem. praecox. Fortschr. d. Med. 34, 201. $-{ }^{6}$ ) Birn ba um, Psychosen mit Wahnbildung und wahnhafte Einbildungen bei Degenerativen. Halle: Marhold 1908. - 7) Birnbaum, Zur Lehre von den degenerativen Wahnbildungen. Allg. Zeitschr. f. Psych. 66, H. 1. - $\left.{ }^{8}\right)$ Birnbaum, Zur Frage der psychog. Krankheitsformen. Zeitschr. f. d. ges. Neurol. u. Psychiatr., Orig., 1. 1910. $\left.{ }^{9}\right)$ Birnbaum, Kriegsneurosen und -psychosen auf Grund der gegenwärtigen Kriegsbeobachtungen. Sammelbericht. Zeitschr. f. d. ges. Neurol. u. Psychiatr., 'Ref. u. Erg., 13, 457; 14, 193 u. 313. - 10) Birnbaum, Dementia praecox und Wahnpsychosen der Degenerierten. Zentralbl. f. Nervenh. u. Psych. 1909, S. 429. - 11) Bleuler, Wahnhafte Einbildungen der Degenerierten. Centralbl. f. Nervenheilk. u. Psych. 1909, S. 77. - 12) Ble uler, Zur Theorie des schizophrenen Negativismus. Psychiatr.-neurol. Wochenschr. 12, 171. 1910. 13) Bonhoeffer, Wie weit kommen psychogene Krankheitszustände vor, die nicht der Hysterie zuzurechnen sind? Allg. Zeitschr. f. Psych. 68, 371. — ${ }^{14}$ ) Bon hoeffer, Die Differentialdiagnose der Hysterie und psychopathol. Konstitution gegenüber der Hebephrenie im Felde. Med. Klin. 1915, S. 877. - 15) Bornstein, Hysterische Psychose mit Verworrenheit und Desorientierung. Jahresber. 1909, S. 1071. - ${ }^{16}$ ) Bu mke, Über die Pupillenstörungen bei der Dementia praecox. Münch. med. Wochenschr. 1910, S. 2688. - 17) B u m ke, Über Pupillenstarre im hysterischen Anfall. Münch. med. Wochenschr. 1906, Nr. 16. 18) B u m ke, Die Pupillenstörungen bei Geistes- und Nervenkrankheiten. Fischer, Jena 1904. - 19) Bumke, Körperliche Symptome der Dementia praecox. Zeitschr. f. d. ges. Neurol. u. Psychiatr., Ref. u. Erg. 14, 303. 1917. — 20 ) D u pré und Gelma, Symptôme de Ganser chez un hébéphrénique. Pariser Psych. Gesellsch. L'encéphale 5, 456. 1910. Ref.: Zeitschr. f. d. ges. Neurol. u. Psychiatr., Ref. u. Erg., 1910. 1, 534. - $\left.{ }^{21}\right)$ Dreyf us, Ein Beitrag zur Kenntnis des hysterischen Irreseins. Centralbl. f. Nervenheilk. u. Psych. 1\%, 785. 1906. 22) Forster, Hysterische Reaktion und Simulation. Monatsschr. f. Psychiatr. u. Neurol. 42, H. 6. 1917. - ${ }^{23}$ ) Friedländer, Die Beurteilung schizophrener Erkrankungen auf Grund der Kriegserfahrungen. Zeitschr. f. d. ges. Neurol. u. Psychiatr., Orig., 43, 301. 1919. - 24) Fuchs, A., Die diagnostische Bedeutung von Pupillenstörungen bei Dementia praecox. Psychiatr.-neurol. Wochenschr. 19, 127, 143, 157. 1917. - 25) Gelant, Die Neologismen der Geisteskranken. Arch. f. Psych. 61. - 26) Gallus, Negat. Erscheinungen bei Geisteskranken und Gesunden. Zeitschr. f. Psychotherap. u. med. Psychol. 5, 327. 1913. — ${ }^{27}$ ) Ganser, Über einen eigenartigen hysterischen Dämmerzustand. Arch. f. Psych. 30, 633. 28) Ganser, Zur Iehre vom hysterischen Dämmerzustand. Arch. f. Psych. 38, 34. - 29) Ga u p p, Zur Frage der katatonischen Psychosen. Centralbl. f. Nervenheilk. u. Psych. 1903. — ${ }^{30}$ ) Halberstadt, G., Contribution à l'étude des psychoses hystérodégénératives. Rev. de Psych., 14, 284. 1910. - ${ }^{31}$ ) Halberstadt, G., Phénomènes hystériformes au début de la démence precoce. Rev. neurol. 18 (2), 161. 1910. - ${ }^{32}$ ) Heilig, Zur Kenntnis der Pathogenese psychogener Dämmerzustände. Arch. f. Psych. 55, 113. - ${ }^{33}$ ) Herzig, Psychosen nach Granatkommotion. Jahrb. f. Psych. 39, H. 2 u. 3. - ${ }^{34}$ ) Isserlin, Über Beurteilung von Bewegungsstörungen bei Geisteskranken. Zeitschr. f. d. 
ges. Neurol. u. Psychiatr., Orig., 3, 511. - ${ }^{35}$ ) Isserlin, Über Jungs ,Psychologie der Dementia praecox" und die Anwendung Freudscher Forschungsmaxismen in der Psychopathologie. Centralbl. f. Nervenheilk. u. Psych. 1907, S. 329. ${ }^{36)}$ Jakob, Kommotionspsychose? Zeitschr. f. d. ges. Neurol. u. Psychiatr. 45, H. 1 u. 2. - ${ }^{37}$ ) J ung, C., G., ťber die Psychologie der Dementia praecox. Halle, Marhold 1907. - ${ }^{38}$ ) Kaiser, Beiträge zur Differentialdiagnose der Hysterie und Katatonie. Allg. Zeitschr. f. Psych. 58, 957; 59, 1H. 1. - ${ }^{39}$ ) Kohnstam m, Schizothymie und Cyclothymie. Berl. klin. Wochenschr. 1914, S. 1680. 40) K napp, Körperliche Symptome bei funktionellen Psychosen. Arch. f. Psych. 44, 709. - 41) Kutner, Über katatonische Zustandsbilder bei Degenerierten. Allg. Zeitschr. f. Psych. 67, H. 3. - 42) Krae pelin, Lehrbuch der Psychiatrie. 8. Aufl. - ${ }^{43}$ ) Kreuser, Zur Differentialdiagnose zwischen Hebephrenie und Hysterie. Allg. Zeitschr. f. Psych. ro, 673. — ${ }^{44}$ ) Lewi n, Ja mes, Ủber Situationspsychosen. Arch. f. Psychiatr. u. Nervenkrankh. 58, 533. - 45) Löwenstein, Boitrag zur Differentialdiagnose des katatonen und hysterischen Stupors. Allg. Zeitschr. f. Psych. 65. - ${ }^{46}$ ) Löwy, Stereotype, pseudokatatone Bewegungen bei leichtester Bewußtscinstrübung (im ",hysterischen" Ausnahmezustand). Zeitschr. f. d. ges. Neurol. u. Psychiatr., Orig., 1, 330. 1910. _- ${ }^{47}$ ) Löw y, Zur Ätiologie psychischer und nervöser Störungen der Kriegsteilnehmer. Monatsschr. f. Psychiatr. u. Neurol. 41, H. 3. - ${ }^{48}$ ) Lückerath, Zur Differentialdiagnose zwischen Dementia praecox und Hysterie. Allg. Zeitschr. f. Psych. 68, 312. $\left.{ }^{49}\right)$ Lührmann, Über die Vortäuschung verschiedener Krankheiten durch Hysterie. Arch. f. Psych. 31, 939. - 50) Matta uscheck, Vorstellung eines Falles von hysterischem Dämmerzustand. Ref. Jahrb. f. Psych. 28, 374. - 51) Me yer, E., Pupillenstörungen bei Dementia praecox. Berl. klin. Wochenschr. 1910, S. 1813. _._ 52) Me yer, E., Bemerkungen zu der Differentialdiagnose der psychogenen Reaktionen mit besonderer Berücksichtigung der im Kriege beobachteten psychischen Störungen. Arch. f. Psych. 56, 246. - ${ }^{53}$ ) Me yer, E., Äußere Einflüsse und psychogene Dämmerzustände. Arch. f. Psych. 60, H. 2 u. 3. -. 54) Me yer, E., Bemerkungen zu Jung: ,Über die Psychologie der Dementia praecox". Arch. f. Psych. 43, 1312. - ${ }^{55}$ ) M ucha, Bemerkungen zur Lehre von der Katatonie. Arch. f. Psych. 31, 928. - 56) Muratow, Zur Nosologie der hysterischen Psychose. Sektion f. Nerven- und Geisteskrankheiten des XI. Pirogoffschen Kongresses in St. Petersburg. Psychotherapie 1, 266. 1910. - ${ }^{57}$ ) Mar kus, Ủber Associationen bei Dementia praecox. Arch. f. Psych. 48, 344. 58) Neisser, Differentialdiagnostische Bemerkungen über das Symptom des Verbigerierens. Allg. Zeitschr. f. Psych. 46, 1890. — ${ }^{59}$ ) Niss1, Hysterische Symptome bei einfachen Seelenstörungen. Centralbl. f. Nervenkrankh. u. Psych. 1902, S. 3. - ${ }^{60}$ ) Pfersdorf, U̇ber Assoziationen bei Dementia praecox. Vortrag. Bericht. Arch. f. Psych. 49, 336. - $\left.{ }^{61}\right)$ Pick, Zum Verständnis des sog. hysterischen Vorbeiredens im hysterischen Dämmerzustand. Monatsschr. f. Psychiatr. u. Neurol. 42, H. 4. 1917. - ${ }^{62}$ ) Piers o n, Zur Differentialdiagnose von Dementia praecox und hysterischen Dämmerzuständen. Inaug.-Diss. Königsberg 1918. ${ }^{63}$ ) Plaskuda, Über Stereotypien und sonstige katatonische Erscheinungen bei Idioten. Zeitschr. f. d. ges. Neurol. u. Psychiatr., Orig., 4, 399. - ${ }^{64}$ ) Pop per, Zur Differentialdiagnose schizophrener und hysterischer Zustandsbilder. Monatsschr. f. Psychiatr. u. Neurol. 46 (4), 362. 1919. - ${ }^{65}$ ) Prengowski, Zur Kenntnis der Krankheitserscheinungen der Nerven, insbesondere über Negativismus und Kigensinn der Degenerierten. Zeitschr. f. d. ges. Neurol. u. Psychiatr., Orig., 38, 165. 1916. - ${ }^{66}$ ) Raecke, Beiträge zur Kenntnis der hysterischen Dämmerzustände. Allg. Zeitschr. f. Psych. 58, 115. - ${ }^{67}$ ) Raecke, Zur Lehre vom hysterischen Irresein. Arch. f. Psych. 40, 171. - ${ }^{88}$ ) Raecke, Utber hy- 
sterische und katatonische Situationspsychosen. Arch. f. Psych. 55, 771. ${ }^{69}{ }^{9}$ Raimann, Die hysterischen Geistesstörungen. Wien-Leipzig, 1904. Ref. im Arch. f. Psych. 39, 949. - ${ }^{70}$ ) Ranninger, Über Sprachstörungen bei Katatonie. Arch. f. Psych. 31, 930. - 71) Reichmann, Frieda, Über Pupillenstörungen bei Dementia praecox. Arch. f. Psych. 53, H. 1, S. 302. - 72) Richter, Kriegsneurosen und Psychogenie. Wien. klin. Wochenschr. 1917, Nr. 52. - ${ }^{73}$ ) Risch, Über die Verkennung psychogener Symptomenkomplexe der frischen Haft und ihre Verwechslung mit Katatonie. Monatsschr. f. Psychiatr. u. Neurol. 25, 291. ${ }^{74}$ ) Risch, Die forensische Bedeutung der psychogenen Zustände und ihre Abgrenzung von der Hysterie. Centralbl. f. Nervenkrankh. u. Psych. 1908, S. 369. - ${ }^{75}$ ) Runge, Pupillenuntersuchungen bei Geisteskranken und Gesunden. Areh. f. Psych. 51, 968. - ${ }^{76}$ ) Schmidt, W., Schizophrenie und Dienstbeschädigung. Monatsschr. f. Psychiatr. u. Neurol. 40, 239. — ${ }^{77}$ ) Schmidt, Die psychischen und nervösen Folgezustände nach Granatexplosionen und Minenverschüttungen. Zeitschr. f. d. ges. Neurol. u. Psychiatr., Orig., 89 (5), $514 . \quad$ - ${ }^{78}$ ) Schneider, Kurt, Über das Wesen und die Bedeutung der katatonen Symptome. Zeitschr. f. d. ges. Neurol. u. Psychiatr., Orig., 22, 486. $-{ }^{79}$ ) Schneider, K urt, Schizophrene Priegspsychosen. Zeitschr. f. d. ges. Neurol. u. Psychiatr., Orig., 43, H. 3 bis 5. - $\left.{ }^{80}\right)$ Schultz, Beiträge zur somatischen Symptomatik und Diagnostik der Dementia praecox. Monatsschr. f. Psychiatr. u. Neurol. 37, 206. _ 81) Se verin, Über Adrenalinwirkung bei Schizophrenen und Gesunden. Monatsschr. f. Psychiatr. u. Neurol. 40, 265. - ${ }^{82}$ ) Siebert, Hysterische Dämmerzustände. Arch. f. Psych. 1919, H. 1. - ${ }^{83}$ ) Stern, Beiträge zur Klinik hysterischer Situationspsychosen. Arch. f. Psych. 50, 640. - 84) Stierlin, Nervöse und psychische Störungen nach Katastrophen. Dtsch. med. Wochenschr. 1911, Nr. 44, S. 2028. 85) Stockmayer, Zur psychologischen Analyse der Dementia praecox. Centralbl. f. Nervenkrankh. u. Psych. 1909, S. 699. - 86) Sträussler, Über den Zustand des Bewußtseins im hysterischen Dämmerzustand. Zeitschr. f. d. ges. Neurol. u. Psychiatr., Orig., 16 3/4 44l. - 87) Strä ussler, Beiträge zur Kenntnis der hysterischen Dämmerzustände. Über eine eigenartige, unter dem Bilde eines psychischen Puerilismus verlaufende Form. Jahrb. f. Psych. 33. - ${ }^{88}$ ) Town se nd, The Ganser symptom complex. Americ. journ. of insanity 66, 631. 1910. $\left.{ }^{89}\right)$ Uhlich, Zur Differentialdiagnose zwischen hysterischen Dämmerzuständen und katatonischer Form der Dementia praecox. Dtsch. militärärztl. Zeitschr. Nr. 24, S. 1017. - ${ }^{90}$ ) Urstein, Die Dementia praecox und ihre Stellung zum manisch-depressiven Irresein. Urban \& Schwarzenberg 1909. - ${ }^{91}$ ) Vogt, Zur Psychologie der katatonischen Symptome. Centralbl. f. Nervenkrankh. u. Psych. 25. 1902. - ${ }^{92}$ ) Vorster, UUber hysterische Dämmerzustände und das Symptom des Vorbeiredens, Monatsschr. f. Psychiatr. u. Neurol. 15, H. 3. ${ }^{93}$ ) Wachs m uth, Beiträge zur Felddiagnose Hysterie. Arch. f. Psych. 52, 993. 94) Weil, Über hysterische Pupillenveränderungen. Arch. f. Psych. 32, 652. ${ }^{95}$ ) Weiler, Untersuchung der Pupille und der Irisbewegungen beim Menschen. Zeitschr. f. d. ges. Neurol. u. Psychiatr. 2, 101. - ${ }^{96}$ ) Westphal, Über Pupillenerscheinungen bei Hysterie. Berl. klin. Wochenschr. Nr. 47, S. 1897. - ${ }^{97}$ ) Westphal, Über Pupillenphänomene bei Katatonie, Hysterie und myoklonischen Symptomenkomplexen. Monatsschr. f. Psychiatr. u. Neurol. 4r (4), S. 187. 1920. - ${ }^{98}$ ) Westphal, Über hysterische Dämmerzustände und das Symptom des Vorbeiredens. Neurol. Centralbl. 1903, Nr. 1. - 99) We igandt, Dementia praecox und Idiotie. Arch. f. Psych. 40, 1051. - $\left.{ }^{100}\right)$ Wilmanns, Zur Differentialdiagnostik der funktionellen Psychosen. Centralbl. f. Nervenkrankh. u. Psych. 1907, S. 569. - 101) Wulff, Beitrag zur Psychologie der Dementia praecox. Centralbl. f. Nervenkrankh. u. Psych. 1909, S. 113. 